\title{
Abstracts for the 37th Human Genetics Society of Australasia Annual Scientific Meeting Queenstown, New Zealand August 4-7, 2013
}

\section{Poster Display}

\section{FOR YOUR INFORMATION? ETHICS AND USING NON-INVASIVE PRENATAL TESTING WITH NO INTENTION TO TERMINATE}

Ainsley Newson' ${ }^{\prime}$, Angus Clarke' ${ }^{2}$ Zuzana Deans ${ }^{3}$

${ }^{1}$ University of Sydney, Sydney, NSW, Australia

University of Cardiff, Cardiff, UK

${ }^{3}$ University of Briston, Bristol, UK

Non-invasive prenatal testing (NIPT) allows women or couples to obtain highly predictive information about the health of a fetus via a blood test during pregnancy. In addition to helping decide how to manage a pregnancy or birth, prenatal testing can also be used to test purely 'for information', that is, the knowledge gained will not determine whether a pregnancy will continue. Given that NIPT can be performed without posing a risk to the pregnancy, and that technology for whole genome sequencing is now technically feasible, we may soon see an increase in requests for NIPT for 'information only' with no intention to terminate the pregnancy based on the results.

In this paper, we examine the ethics of using NIPT 'for information' only. We discuss competing interests and the acceptability of testing for 'frivolous' reasons. Working from the presumption that NIPT for information only is acceptable in at least some circumstances, we claim that arguments about testing children for genetic conditions are relevant to determining how NIPT should be used for information only. We argue that providing information about adult-onset conditions will usually be inappropriate. We also discuss the potential objectification of the future child. However, a context-based decision, accounting for competing interests, may be required when determining whether to provide information about childhood onset conditions, non-medical traits or carrier status. In some circumstances, it may be appropriate to counsel against or even deny access to information, justified by the impact testing is likely to have on the future child.

\section{SEVERE THROMBOSIS IN A 20-YEAR-OLD FEMALE WHO IS A COMPOUND HETEROZYGOTE FOR MUTATIONS IN THE PROTEIN C GENE: A CASE REPORT}

Alicia Byrne, Alexandra Jolley, Nancy Lerda, Catherine Nicholls, David Ross, Magdalena Sobieraj-Teague, Susan Rodgers, Simon McRae, Hamish Scott

SA Pathology, Adelaide, SA, Australia

Background: Hereditary protein $\mathrm{C}$ deficiency has been described as having autosomal dominant and autosomal recessive inheritance
(OMIM phenotypes 612304 and 176860), possibly reflecting a functional interaction with other genes in the clotting cascade that has not been clearly elucidated. The incidence of asymptomatic protein $\mathrm{C}$ deficiency has been reported to be $1 / 500$ healthy individuals, whereas the incidence of clinically significant protein $\mathrm{C}$ deficiency is estimated at $1 / 20,000$. The objective of the current study was to elucidate the molecular basis of the Protein C deficiency in a family where the proband presented at an early age with thromboembolitic episodes, then at 20 years of age with severe thrombosis. Coagulation studies revealed Protein $\mathrm{C}$ deficiency, with activity measured at $11 \%$ (RR 65-130\%). Methods: The entire coding region, associated splice junctions and promoter region of the Protein $\mathrm{C}$ gene (PROC) in the proband, her parents and her half brother were analysed by sequence analysis. Results: The proband was found to be compound heterozygous for a previously undescribed frameshift mutation, c.388-397del in exon 5 and a missense mutation, c.541T $>\mathrm{C}$ in exon 7 of PROC. Both parents were asymptomatic heterozygotes. Conclusion: A clear correlation between genotype and phenotype in protein $\mathrm{C}$ deficiency has been difficult to establish as the result of conflicting reports in the literature. This case highlights the need for a better understanding of the genotype phenotype correlation in hereditary Protein $\mathrm{C}$ deficiency and further comprehension of the functional interaction of genetic variation in the many different components of the coagulation cascade.

\section{3. 'THAT'S JUST THE WAY IT IS': EXPLORING THE USE OF GENETIC SERVICES BY RURAL AND REGIONAL CLIENTS IN VICTORIA}

Alisha McLauchlan', Sylvia Metcalfe' , Jane Wallace ${ }^{2}$, Mary-Anne Young ${ }^{3}$, Belinda McClaren', Alice Weeks'

${ }^{1}$ Murdoch Childrens Research Institute, Melbourne, VIC, Australia

${ }^{2}$ Victorian Clinical Genetic Service, Melbourne, VIC, Australia

${ }^{3}$ The Peter MacCallum Familial Cancer Centre, Melbourne, VIC, Australia

This study explored the use of genetic services by rural and regional clients in Victoria; with the aim to gain insight into any potential issues these clients face when accessing genetic services. This study utilized a qualitative approach with the conceptual framework of program evaluation. Twelve participants were recruited from two clinical genetic services: The Victorian Clinical Genetics Service (VCGS) and The Peter MacCallum Familial Cancer Centre (FCC). 
This study has revealed that participants are generally satisfied with the service and care they received from regional genetics clinics. Participants were grateful for the option to be seen closer to their home location. Although satisfied with the service, participants did identify a number of barriers that make genetics services less accessible for rural and regional clients compared to metropolitan populations, including travel, travel costs and the lack of awareness of the service. Participants reported that accessibility issues for genetic services were similar to other regional health care services. Participants felt that there were health care disadvantages to living in rural and regional areas of Victoria compared to metropolitan cities, but believed that this was simply a consequence of living in the country.

This is the first study to examine how rural and regional clients use genetic services in Victoria. It has provided a foundation to further explore best practice for genetic services and how these services can adapt in order to best meet the needs of rural and regional clients.

\section{A RARE FINDING OF TRIPLE HIT LYMPHOMA - CASE REPORT}

Anne Jay, Richard Parfitt, Angela Brown, Robert Weinkove

Capital and Coast DHB, Wellington, New Zealand

Double hit lymphoma accounts for up to $12 \%$ of Diffuse Large Bcell Lymphoma (DLBCL) cases. These cases show rearrangements of both the MYC and the BCL2 loci, and have a very poor prognosis. They are characterized by highly aggressive clinical behavior, and pathological features overlapping with Burkitt lymphoma, diffuse large B-cell lymphoma and B-lymphoblastic lymphoma/leukaemia.

We present here a rare finding of a triple hit lymphoma. Our patient, a female aged 66 years, was referred for cytogenetic testing in April 2012. She was a known DLBCL patient, and a bone marrow sample was received for staging.

G-banded karyotyping revealed a complex karyotype, including a $t(3 ; 22)$ translocation, a complex rearrangement involving both a $\mathrm{t}(14 ; 18)$ and $\mathrm{t}(8 ; 14)$ and other structural and numerical abnormalities. The involvement of the MYC (8q24), BCL2 (18q21)and BCL6 (3q27) genes was confirmed by FISH using the Vysis break apart probes for these 3 loci.

A review of 7 cases of triple hit lymphoma suggests that these cases have a worse prognosis than double hit lymphoma, showing a shorter overall survival with a median survival time of 4 months.

\section{AN INTRAGENIC DELETION IN THE NFIA GENE IN A PATIENT WITH A HYPOPLASTIC CORPUS CALLOSUM, CRANIOFACIAL ABNORMALITIES AND URINARY TRACT DEFECTS}

Anupam Rao', Sheridan O'Donnell ${ }^{2}$, Nicole Bain ${ }^{3}$, Damon Shorter ${ }^{4}$, Himanshu Goel $^{2}$

\footnotetext{
${ }^{1}$ University of Newcastle, Newcastle, NSW, Australia

${ }^{2}$ Hunger Genetics, Newcastle, NSW, Australia

${ }^{3}$ Hunter Area Pathology Service, Newcastle, NSW, Australia

${ }^{4}$ General Pediatrician, Australia
}

Background: Chromosome 1p31 deletion syndromes affecting the NFIA gene are a rare set of conditions characterized by variable defects in formation of corpus callosum, craniofacial abnormalities and urinary tract defects. A review of current literature suggests only seven cases have been reported, none of which had an isolated NFIA gene defect. Methods: We submit the clinical and molecular features of an 8-year-old female patient with microdeletion of chromosome 1p31.1 who has developmental delay, macroscopic hemoglobinuria and metopic synostosis. She was investigated with karytopying, subtelomeric FISH and microarray CGH. Results: Array CGH identified a single $116.50 \mathrm{~kb}$ microdeletion of $1 \mathrm{p} 31.1$ involving exons 4-9 of the NFIA gene. Her MRI Brain showed hypoplasia of the corpus callosum especially in the posterior areas. Karyotype was normal, ruling out structural chromosomal abnormalities. Conclusion: In this study, we showed that an intragenic deletion in the NFIA gene is associated with hypoplasia of the corpus callosum, intellectual disability, metopic synostosis and urinary tract abnormalities. Furthermore, we also propose a mechanism by which disruptions in the NFIA gene causes craniofacial abnormalities. This report presents the first case of an intragenic deletion within the NFIA gene that is still consistent with classic clinical phenotypes present in previously reported cases of chromosome $1 \mathrm{p} 31.3$ related deletion. This finding will help clarify the role of the NFIA gene in the normal formation of parts of the CNS, the craniofacial complex and the urinary tract.

\section{REFERRING PATIENTS FOR PREIMPLANTATION GENETIC DIAGNOSIS - A SURVEY OF CLINICIANS.}

April Morrow', Janan Karatas ${ }^{2}$, Sean Seeho ${ }^{3}$, Kristine Barlow-Stewart ${ }^{3}$, Bettina Meiser $^{4}$

1 University of Sydney, Sydney, NSW, Australia

${ }^{2}$ Neuroscience Research Australia, Sydney, NSW, Australia

${ }^{3}$ Sydney Medical School - Northern, Sydney, NSW, Australia

${ }^{4}$ Psychosocial Research Group, University of New South Wales, Sydney, NSW, Australia

Introduction: Preimplantation genetic diagnosis (PGD) is an assisted reproductive technique in which embryos are tested for specific genetic abnormalities to enable the selection of only unaffected embryos for implantation and pregnancy. This provides an alternative to prenatal diagnosis and potential termination of pregnancy for couples at risk of transmitting a serious genetic disorder to their children. A recent study conducted by members of the investigative team (Barlow-Stewart, Meiser, Karatas) identified individuals who had not been referred by their obstetrician to PGD and felt disempowered and angry at not being given this option. This study aims to identify the barriers to referral and attitudes of obstetricians and gynaecologists to PGD. Methods: An online questionnaire was distributed by e-mail to registered members of The Royal Australian and New Zealand College of Obstetricians and Gynaecologists (RANZCOG). The survey explored participant's professional background, knowledge of PGD and perceived barriers to referral for PGD. Results: 365 responses were obtained (86\% RANZCOG Fellows, $14.2 \%$ Accredited RANZCOG trainees, mean age range 41-50 years). Of these, 228 (58\%) had referred patients for consideration of PGD as a reproductive option. There was a tendency to underestimate the cost of PGD per cycle, with $260(66 \%)$ believing the out-of-pocket expense to be less than \$9,000AUD. 155 (42\%) and $143(39 \%)$ participants reported availability/access to PGD services and patient financial status (respectively) to be either 'mostly' or 'always' barriers to referral. This is an ongoing study and data analysis is continuing. Conclusions: Our preliminary data identifies existing barriers that thwart communication about PGD between obstetricians and their patients. Such barriers guide suggestions for future education of these health professionals as well as marketing of the availability of this technique.

\section{THE EVOLUTION OF MOLECULAR DETECTION TECHNIQUES OVER 30 YEAR IN CLINICAL GENETICS LABORATORY, MONASH MEDICAL CENTRE, VICTORIA, AUSTRALIA}

Asif Alam' ${ }^{\prime}$ Kerryn Weekes ${ }^{2}$, Ruoxin $\mathrm{Li}^{2}$, Jeremy Wells ${ }^{2}$, Carly Wishart ${ }^{2}$, Clyde Henriques $^{2}$, Elizabeth Algar ${ }^{2}$, Donald K Bowden ${ }^{2}$

${ }^{1}$ Monash Health, Melbourne, VIC, Australia

${ }^{2}$ Clinical Genetics Laboratory, Monash Health Clayton (MMC), Monash Health, Melbourne, VIC, Australia

The Clinical Genetics Laboratory, Monash Medical Centre, Monash Health, Clayton, Victoria, Australia, a state-funded service, has been performing molecular detection of thalassaemias and other 
hemoglobinopathies, hemophilia A and B, and hemochromatosis for more than two decades. Testing for alpha thalassaemia by DNA analysis began in Victoria in 1983 using Southern analysis. Since then, the laboratory has expanded and incorporated various techniques including Gap-PCR, Sanger Sequencing, Multiplexed Ligation Dependent Probe Amplification and Next Generation Sequencing using Ion Personal Genomic Machine, $\tilde{N} \notin$ (PGMÑ $\phi$ ) to accommodate increased sample numbers, diversity and complexity of genotypes from a very diverse population, to encompass rarer mutations and to increase throughput using cutting edge technologies.

We have identified many rare mutations, both published and unpublished over the years. Collectively these mutations have contributed considerably in clarifying the phenotypic expression of some of these rare mutations. The laboratory has employed collective data and experience to develop screening protocols for thalassaemias and hemoglobinopathies and has developed robotic technologies to increase PCR throughput. Recently, the Ion Personal Genomic Machine, $\tilde{\mathrm{N}} \notin(\mathrm{PGMN} \notin)$ has been employed in our laboratory to reliably identify FVIII mutations.

We present here clinical genetic laboratory work-flow, share our experience with the newer methods, present frequency of detection of previously undescribed mutations and rare mutations and some interesting cases.

\section{HOW MUCH PROTEIN CAN HE TOLERATE? A STUDY OF PHENYLALANINE TOLERANCE IN A BOY WITH PHENYLKETONURIA}

Barbara Dennison, Susan Thompson, John Christodoulou

The Children's Hospital at Westmead, Sydney, NSW, Australia

The parents of a 2-year-old boy diagnosed at birth with phenylketonuria (PKU) initiated the discussion on introducing tetrahydrobiopterin (BH4) to his management. On day 17 of life he was shown to be BH4-responsive on a BH4 load. BH4 is not available to PKU patients in NSW. His grandmother was prepared to fund the estimated $\$ 30,000$ p.a. cost of BH4.

His usual PKU diet provided $34 \mathrm{mg}$ Phe/kg/day. To study his Phe tolerance and $\mathrm{BH} 4$ requirement, he was given additional Phe (provided as a free amino acid added to his formula), $50 \mathrm{mg} / \mathrm{kg} /$ day initially and a week later $100 \mathrm{mg} / \mathrm{kg} / \mathrm{day}$, divided into three doses daily. Weekly decreasing doses of BH4 $(20 \mathrm{mg}, 10 \mathrm{mg}, 5 \mathrm{mg} / \mathrm{kg} /$ day $)$ were given as a twice a day dose. His daily Phe levels remained within the therapeutic target range (100-350 umol/l) irrespective of Phe intake and $\mathrm{BH} 4$ dose. He continued to take $1.6 \mathrm{~g}$ protein equivalent from his PKU supplement. Without $\mathrm{BH} 4$ and with additional Phe equivalent to the protein intake of a similar-aged child without PKU eating meat, dairy and normal cereals, his blood Phe levels remained within target range. His parents were then encouraged to liberalise his protein intake by using normal bread, rice and pasta and some dairy foods.

Conclusions: This child is able to tolerate significantly more Phe than initially thought. The increase in food protein has greatly improved his quality of life. Our experience highlights the importance of establishing Phe tolerance prior to commencing BH4, and underscores the need to develop a structured approach to this.

\section{PREDICTORS AND BARRIERS FOR INFORMATION SHARING BETWEEN CF CARRIERS AND THEIR FAMILIES}

Carolyn Shalhoub', Melanie Leffler ${ }^{2}$
${ }^{1}$ Sydney Children's Hospital, Sydney, NSW, Australia
${ }^{2}$ Royal North Shore Hospital, Sydney, NSW, Australia

Cystic fibrosis (CF) is a life-limiting, autosomal recessive genetic condition, with one in 25 individuals of Caucasian ancestry being carriers. Identification of a CF-affected individual in a family can have significant implications for their obligate carrier parents and the extended family, who are at an increased risk of being CF carriers and having children affected with $\mathrm{CF}$. The extent to which carriers share relevant genetic information with their at-risk family members is of significance in order for their relatives to make informed decisions about genetic testing and reproductive options. The aims of this study were to elucidate potential barriers and predictors of disclosure in CF obligate carriers frequenting the Sydney Children's Hospital CF clinic through an exploration of both the pattern of information dissemination and reported reasoning for their disclosure.

A mixed-surveying method approach was utilized, including a questionnaire and interview. Positive correlations were identified for participants' reported emotional relationship with their relatives, the female gender and perceptions of their relatives' risk of being a carrier. Negative correlations included participant education level, child's diagnosis age and time since initial CF diagnosis. Reasons for high disclosure included desiring their relatives to understand their genetic status, reproductive implications of being a carrier, support and concern from relatives, and strong emotional connections. Reasons for low disclosure included disinterest, perceived irrelevancy of information for their relatives, and emotional disconnect.

This research highlights important factors genetic counselors may explore with clients, following genetic test results that may have significant implications for clients' families.

\section{TOWARDS REDUCING DECISIONAL UNCERTAINTY DURING PRENATAL GENETIC TESTING DECISION-MAKING}

\section{Cecile Muller' ${ }^{\prime}$ Linda Cameron ${ }^{2}$}

${ }^{1}$ Murdoch Children's Research Institute, Melbourne, VIC, Australia

${ }^{2}$ University of California, Merced, CA, USA

Prenatal diagnostic tests (e.g., amniocentesis) can determine whether a fetus carries a genetic abnormality. However, these invasive procedures can also result in pregnancy loss. Given that prenatal diagnostic tests have no obvious pros or cons, the decision about whether or not to undergo the procedure is likely to be a difficult one, and to elicit a state of psychological struggle known as decisional uncertainty. This study examined predictors of prenatal testing decisional uncertainty. Over 450 New Zealanders (345 women; $M$ age $=32.68$ years; $S D=8.61$ ) read a hypothetical scenario asking them to imagine they expected a child and had heard about a pre-birth test that could detect fetal abnormalities responsible for deteriorations of the cognitive, neurological and emotional systems. Participants then completed measures of condition coherence (i.e., how much the condition under investigation made sense to them), child-related worry, motivation to comply with doctors' perceived preferences, perceived benefits from receiving normal results, and perceived differences of opinions from partner's preferences regarding prenatal testing. Results from regression analyses showed that greater decisional uncertainty was predicted by lower condition coherence $(\beta=-0.13)$, greater childrelated worry $(\beta=0.17)$, greater motivation to comply with doctors' perceived preferences $(\beta=0.22)$, greater perceived benefits from receiving normal results $(\beta=0.11)$, and greater differences in opinions from partner's perceived preferences $(\beta=$ $0.18)$. In conclusion, decisional uncertainty could be minimized and important reproductive decision-making facilitated if concerns related to family planning are identified and appropriately addressed. 
11. 'IGNORANCE IS BLISS': THE EXPERIENCE OF PARENTS FROM THE RUBINSTEIN TAYBI SYNDROME AUSTRALIA SUPPORT GROUP ON WAITING FOR A DIAGNOSIS

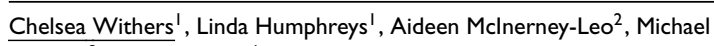
Gabbett $^{3}$, Jane Flemming ${ }^{\prime}$

${ }^{1}$ Griffith University, Brisbane, QLD, Australia

${ }^{2}$ University of Queensland, Brisbane, QLD, Australia

${ }^{3}$ Genetic Health Queensland, Brisbane, QLD, Australia

Background: Diagnosis of genetic conditions can be challenging due to their rarity and the fact that identifiable features may not be evident at birth. Both of these challenges apply in Rubinstein-Taybi syndrome (RTS), and for parents this can mean living in a state of uncertainty. The aim of this study was to identify common factors associated with the psychosocial impact of a delayed diagnosis. Methods: Parents of the Rubinstein Taybi Syndrome Australia support group, who have children with RTS, were invited to participate in this study. A qualitative, semi-structured interview was developed and administered. Telephone interviews were then transcribed, deidentified and analyzed for recurrent themes. Results: A total of 13 individuals, representing 11 of the 40 families known to the support group, agreed to participate. Ages at diagnosis ranged from birth to 3 years (average age 8 months). Prior to diagnosis, parents reported either an 'ignorance is bliss' reaction or a frustration at knowing there was 'something wrong'. They identified the primary benefit of diagnosis was the provision of a 'label' and validation of concerns. Uncertainty was present both pre and post diagnosis. Conclusion: Parents reported a delay in diagnosis can be perceived as beneficial in the short term, as a period of normality allowed for bonding, but frustrating in the long term as parents had a growing sense of there being 'something wrong'. The desire to end uncertainty drove the search for a diagnosis, but many reflected that the uncertainty remained post diagnosis, whilst acknowledging that it was somewhat changed.

\section{MODELING HUMAN MUTATIONS IN KCNT1 CAUSING} EPILEPSY, ID AND PSYCHIATRIC FEATURES IN DROSOPHILA

Chiao Xin Lim, Sarah Heron, Michael Ricos, Tetyana Shandala, Leanne Dibbens

University of South Australia, Adelaide, SA, Australia

Epilepsy is the most common debilitating neurological condition, with a worldwide prevalence of around $2 \%$. More than half of the causes are considered 'idiopathic', where they are mainly genetic in origin. Ion channels mutations are the major cause of seizure phenotypes. Mutations in the potassium channel genes KCNQ2 and KCNQ3 contribute to severe forms of neonatal and childhood-onset focal epilepsies. Our group has found that KCNT1 mutations cause a severe form of autosomal dominant nocturnal frontal lobe epilepsy (ADNFLE), which includes intellectual disability (ID) and psychiatric features. KCNT1 encodes a sodium-activated potassium channel subunit, which is thought to function in ion conductance and developmental signaling pathways. We are currently determining whether KCNT1 mutations also contribute to sporadic cases of focal epilepsy with and without ID and psychiatric features. We are using high resolution melt curve as a powerful analysis tool to look for mutations in 400 sporadic cases of focal epilepsy. To gain insights into the seizure phenotype caused by KCNT1 mutations and to analyze any changes in the morphology of the brain, we are employing Drosophila melanogaster as a model organism. We will use antisense RNA to knockdown KCNT1 expression in this classic genetic model. Both functional studies in Drosophila and identifying new KCNT1 mutations in patients with focal seizures will help us to uncover the mechanisms behind how KCNT1 mutations result in focal seizures, and may provide a seizure model for testing therapeutic interventions.
13. NEW SYNDROME OF ECTRODACTYLY AND LETHAL PULMONARY ACINAR DYSPLASIA ASSOCIATED WITH HOMOZYGOUS FGFR2 LOF MUTATION IDENTIFIED BY EXOME SEQUENCING

Christopher Barnett ${ }^{1,2}$, Nathalie Nataren ${ }^{2}$, Chris Hahn², Damien Bruno ${ }^{3,2}$, Andreas Schreiber ${ }^{2,4}$, Jinghua Feng ${ }^{2,4}$, Andrew McPhee ', Hamish Scott ${ }^{2}$

${ }^{1}$ Women's and Children's Hospital, Adelaide, SA, Australia

2 SA Pathology, Adelaide, SA, Australia

${ }^{3}$ Murdoch Children's Research Institute, Melbourne, VIC, Australia

${ }^{4}$ University of Adelaide, Adelaide, SA, Australia

Ectrodactyly/split hand-foot malformation is genetically heterogeneous with $>100$ syndromic associations. Acinar dysplasia is a rare congenital lung lesion of unknown etiology, which is lethal postnatally. Rare familial cases suggestive of recessive inheritance have been described. Here we present an infant from a consanguineous union with a unique syndrome with both ectrodactyly and acinar dysplasia.

The female infant was born at term but died of severe respiratory failure on day 2. Autopsy confirmed widespread acinar dysplasia. Ectrodactyly involving both hands and feet was observed. The infant also had small ears and atretic external auditory canals.

SNP array and whole exome sequencing identified 22 homozygous candidate causative sequence variants (SV), which with filtering on, prediction of pathogenicity, protein conservation and lung and limb expression, point to only one candidate gene, FGFR2. Molecular modeling suggests this FGFR2 missense variant (R255Q in the IgIII domain (D3) is a loss-of-function (LOF) mutation. Both parents of the affected infant were heterozygous for this mutation and had no phenotypic features.

A knock-in Crouzon Syndrome mouse with a recurrent autosomal dominant activating FGFR2 mutation W290R, also in D3, has been generated. While heterozygous mice show craniofacial malformations and craniosynostosis, homozygous mice also showed limb and organ (including lung) agenesis and died after birth from respiratory failure. The combination of ectrodactyly and acinar dysplasia has not been described before and we propose it represents a new syndrome caused by homozygous LOF FGFR2 mutations.

\section{CHRONIC HISTIOCYTIC INTERVILLOSITIS: AN IMPORTANT CAUSE OF SEVERE FETAL GROWTH RESTRICTION THAT MAY MIMIC BONE DYSPLASIA}

Christopher Manning ', Chris Barnett' , Lynette Moore ${ }^{2}$, Nick Manton ${ }^{2,1}$, J Kaye ${ }^{3}$, Eric Haan'

${ }^{\prime}$ SA Clinical Genetics Service, SA Pathology, Adelaide, SA, Australia

2 Department of Histopathology, Women's and Children's Hospital, Adelaide, SA, Australia

${ }^{3}$ Department of Medical Imaging, Women's and Children's Hospital, Adelaide, SA, Australia

Chronic histiocytic intervillositis (CHIV) is a rare placental condition associated with pregnancy loss, intrauterine growth restriction (IUGR) and high recurrence risk. Placental histology shows an inflammatory process with histiocyte infiltration and fibrin deposition. The etiology and fetal impact of CHIV is poorly understood.

A 28-year-old woman, G3P1, presented with increased Down syndrome risk (markedly low PAPP-A) on first trimester screen. She had one previous normal pregnancy and one early miscarriage. Ultrasound at 20 weeks gestation showed marked IUGR and anhydramnios; the pregnancy was terminated at 27 weeks. Post-mortem examination was suggestive of osteogenesis imperfecta (OI) because of the presence of multiple healing fractures of all long bones and poor bone mineralization. The placenta was extremely small and histopatholgy was consistent with CHIV. Sequencing of the COL1A1, COL1A2, CRTAP and LEPRE1 genes was negative. The possibility of autosomal recessive OI was discussed. 
The couple's next pregnancy resulted in a termination at 21 weeks for severe IUGR accompanied by anhydramnios. Autopsy showed marked shortening of the long bones and head/body disproportion but there were no fractures. Histology of the extremely small placenta again showed CHIV. Treatment with steroids and aspirin in her next pregnancy was associated with live birth at 32 weeks, the neonate having significant asymmetric growth restriction but no fractures. It is unclear whether the bone changes are due to molecularly unproven OI or are an effect of CHIV but this report raises the possibility of CHIV as a cause of a severe OI-like phenotype in the fetus.

\section{AN ASSOCIATION STUDY OF GLUTAMATERIC AND RNA EDITING GENES IN MIGRAINE}

\section{Claudia Gasparini, Lyn Griffiths \\ Griffith University, Brisbane, QLD, Australia}

Migraine is a complex neurological disorder with a strong genetic basis. Approximately $\sim 12 \%$ of the Caucasian population are affected, including $18 \%$ of adult women and $6 \%$ of adult men. This disorder is extremely debilitating with wide-ranging socioeconomic impact that significantly affects both families and employers. Migraine is poorly treated and at present there is no evident biochemical marker or diagnostic test. The aim of this study was to investigate if polymorphisms in the glutamate receptor genes GRIA2 and GRIA4 and variants in two RNA editing genes, ADARB1 and ADARB2 are associated with migraine pathogenesis and susceptibility in an Australian case-control population. The GRIA genes are involved in binding glutamate an excitatory neurotransmitter implicated in migraine processes including cortical spreading depression, trigeminovascular activation and central sensitization. The ADARB1 gene is of particular interest and was chosen for investigation in this study due to its editing function on glutamate and serotonin receptor transcripts. The ADARB2 gene was selected based on significant results of a pGWAS of the isolated population of Norfolk Island. ADARB1 and ADARB2 are RNA editing genes which have been suggested as candidates for complex neurological disorders such as epilepsy, depression and schizophrenia. The results of this study did not show significant association between the tested GRIA gene variants and ADARB2 in migraine. We are currently genotyping further variants in both the ADARB2 and ADARB1 gene by Sequenom to ascertain their potential significance in migraine etiology.

\section{AN IMPROVED METHOD FOR FRAGILE $X$ TESTING - $\mathbf{A}$ REVIEW OF AMPLIDEX ${ }^{\text {TM }}$ GENE-SPECIFIC (GS) FMR1 PCR IN OUR LABORATORY}

\begin{abstract}
Corrina Walsh, George Elakis, Jerry Van Rheeden, Maroulis Sarah, Tran Anh, Melanie Galea, Glenda Mullan, Peter Taylor, Melody Caramins, Michael Buckley South Eastern Area Laboratory Services, NSW, Australia
\end{abstract}

Fragile XA associated disorders are caused by unstable trinucleotide (CGG) repeat expansions in the 5' untranslated region of the FMR1 gene. Fragile XA syndrome is the most common cause of inherited intellectual disability, with the majority of cases associated with a mutation of $>200$ CGG repeats of FMR1. Fragile X-associated tremor/ataxia syndrome and primary ovarian insufficiency occur as a result of FMR1 premutation alleles (55-200 repeats). Traditionally, the molecular diagnosis of FMR1 triplet repeat expansions larger than 75 repeats has relied on Southern blotting. Southern blotting is an expensive and laborious technique requiring DNA of high quantity and quality taking often weeks to complete. In 2010, Asuragen released a PCR-based kit for the reliable detection of the full mutation spectrum of alleles of FMR1, AmplideX $®$ Gene-Specific FMR1 PCR. Here we report our laboratory experience with this kit in the testing of 1,671 postnatal and 9 prenatal samples. We have found the kit to reliably detect mutations across all FMR1 mutation ranges using as little as $40-80 \mathrm{ng}$ of DNA, and allowing for reporting in as few as 2 working days. While the high sensitivity of the assay is advantageous, in that it is robust in requirements of DNA and detection of mosaicism, this renders the technique sensitive to maternal contamination in the prenatal setting, necessitating the utilization of linkage data to verify these results. The implementation of the AmplideX $\otimes$ FMR1 PCR has reduced the average turn-around time for Fragile XA testing from approximately 21 to just 9 days, significantly improving the service provided by our laboratory.

\section{THALASSEMIA GENETIC TESTING: A QUEENSLAND} PERSPECTIVE

\section{David Fairbairn}

Pathology Queensland, Brisbane, QLD, Australia

Alpha and beta thalassemia genetic testing by Pathology Queensland was introduced in 2009 and 2011, respectively. This forms an important part of hemoglobinopathy testing in the pre-pregnancy and antenatal setting for Queensland patients. An algorithm was prepared to ensure that samples met genetic testing criteria so that DNA analysis was only performed when appropriate. In addition to the common large alpha globin deletions that were detected, a copy number variant (CNV) for two contiguous MLPA probes between HBZ and HBZP1 genes on the alpha globin gene cluster at 16p13.3 was observed. The significance of this CNV during MLPA analysis of alpha thalassemia is that it may confound the identification of the type of alpha globin two-gene deletions on the same allele, that is, alpha zero thalassemia. This and other examples highlighted the need for employing more than one detection method to verify or distinguish common large deletions in alpha globin to ensure an accurate determination of genotype. The alpha and beta globin mutation spectrum of Queensland patients will be presented.

\section{UNMET NEEDS IN THE RARE DISEASE COMMUNITY}

Dianne Petrie' ${ }^{\prime}$, Catherine Spinks', Bronwyn Burgess ${ }^{2}$

${ }^{1}$ The Association of Genetic Support, Sydney, NSW, Australia

${ }^{2}$ Hunter Genetics, Newcastle, NSW, Australia

Living with a rare disease and/or caring for someone with a rare disease is not only a financial burden to families, but is associated with significant social and emotional impacts. Families are faced with difficulties in finding a diagnosis and coordinating treatment options for conditions that are mostly chronic, complex, and associated with intellectual, physical or neurological disabilities. Feelings of loneliness and social isolation are compounded by lack of appropriate community support. This exploratory study aims to describe experiences and identify service needs of families and stakeholders affected by rare diseases ( $80 \%$ of which are genetic in origin) in the Hunter Region of New South Wales. Interviews and focus groups will be conducted with volunteer participants who have a rare disease, their family members and loved ones, and service providers involved with care and support (e.g., geneticists, genetic counsellors, allied health professionals, support groups). Grounded in a person-centered approach, the purpose of this study is to give voice to individuals in the rare disease community, to illustrate the current issues and identify possible solutions for government consideration.

\section{FEINGOLD SYNDROME IN SOUTH AUSTRALIA}

Elizabeth Thompson ', Eric Haan', Lesley McGregor', Christopher Barnett ${ }^{2}$, Anne Baxendale

I SA Pathology, Adelaide, SA, Australia

${ }^{2}$ Women's and Children's Hospital, Adelaide, SA, Australia

Twelve individuals with Feingold syndrome from six unrelated families are presented. The hallmark of this autosomal dominant 
disorder is intestinal atresia, often involving the duodenum or esophagus. The penetrance of intestinal atresia is variable, and its presit was then recognized that a parent (with no history of intestinal atresia) had other features such as the characteristic hand and foot abnormalities, microcephaly and short stature. All affected individuals had at least some elements of the characteristic facial appearance due to microcephaly, short upslanting palpebral fissures, short philtrum, down-turned corners of the mouth and micrognathia, often giving a recognisable 'gestalt'. Intellectual disability was present in 3 of the 5 probands who were old enough to be assessed. It was borderline in one, mild in another and unusually for this condition it was severe in the third.

Feingold syndrome is caused by mutations of the MYCN gene on chromosome $2 \mathrm{p} 24$. Two thirds of individuals have a sequence variant and $10 \%$ have a complete or partial deletion. In our families, two had pathogenic sequence variants, one had a deletion of the gene detected on a targeted chromosome array, no sequence or copy number variants were identified in another family in which the affected child had a more severe phenotype, and testing was not done in two families. There is genetic heterogeneity; so called Feingold syndrome 2 is caused by a hemizygous deletion of the MIR17HG gene on chromosome 13q31.3. ence led to the diagnosis in all our probands. In three families,

\section{ORAL HAEMORRHAGIC BULLAE IN EHLERS-DANLOS SYNDROME: AN UNUSUAL PRESENTATION}

Elizabeth Ellis', Mark Schifter ${ }^{2}$, Felicity Collins'

${ }^{1}$ The Children's Hospital at Westmead, Sydney, NSW, Australia
2 Westmead Hospital, Sydney, NSW, Australia

Hemorrhagic bullae of the oral mucosa are possibly a nonspecific finding in several forms of Ehlers-Danlos syndrome (EDS) as sequelae of increased tissue fragility. Oral hemorrhagic bullae have been reported as an early manifestation in a fatal case of EDS type IV - vascular type. We report a case of a 30-year-old female with childhood-onset polyarthralgia, joint hypermobility, finger dislocations and fatigue who presented with a 2-year history of oral hemorrhagic bullae. The bullae predominantly affect the lateral aspects of the tongue and buccal mucosa. They are precipitated by eating even soft foods, with some improvement with pureed diet. The bullae can be $2-3 \mathrm{~cm}$ in size and burst spontaneously, usually after 1-2 hours, to leave an ulcer which heals over 2 days. She has recently developed mild hemorrhagic petechiae of the anterior hard palate. There is a 1-year history of possible autonomic symptoms including dizziness and altered bowel habit. Oesphagogastroduodenoscopy and colonoscopy were unremarkable. The proband's skin bruises easily but scars normally. Hematological investigations were normal apart from borderline low Von Willebrand factor studies. The proband's eldest daughter, aged 5 years, has joint hypermobility, as does as a paternal aunt. There is no other relevant family history. While her clinical presentation suggests EDS type III - hypermobile type, the association of oral haemorrhagic bullae with other forms of EDS, including as a reported prelude to EDS type IV, warrants further investigation. COL3A1 gene testing is in progress to assess for molecular evidence of EDS type IV.

\section{TESTING FOR AORTOPATHIES AND RELATED DISORDERS}

Elizabeth Ormshaw, Katherine Holman, Karen Wong, Lesley Ades, Thet Gayagay, Gladys Ho, Bruce Bennetts

The Children's Hospital at Westmead, Sydney, NSW, Australia

The number of genes associated with aortic dilatation and dissection is expanding. Given that the consequences of aortic dissection are often fatal there is a strong need to determine the underlying etiology, particularly for the extended family as many of the genes involved have an autosomal dominant mode of inheritance. Many of the genes associated with these disorders are involved in the TGFbeta signaling pathway. There are classical clinical phenotypes associated with some of the genes in this pathway, such as Marfan syndrome (FBN1), Loeys Dietz syndrome (TGFBR1\&2) and aneurysm-osteoarthritis syndrome (SMAD3). However, there is considerable phenotypic overlap and knowing which gene to start testing is not always clear cut.

Our laboratory initially established testing for FBN1 and later TGFBR1\&2. Over time, many of the FBN1 and TGFBR1\&2 negative patients have required testing of multiple genes involved in the TGFbeta signaling pathway. This has generally been performed by testing each gene individually and consecutively which is slow and expensive. We are developing and validating a massive parallel sequencing (MPS) strategy for an aortopathy panel of genes (FBN1, TGFBR1, TGFBR2, TGFB2, ACTA2, SMAD3, COL3A1, EFEMP2, FBN2, MYH11, NOTCH1, SLC2A10 and SKI) and initial data will be presented.

We have expanded our Sanger sequencing testing to include the FBN1, TGFBR1, TGFBR2, TGFB2, ACTA2, SMAD3, SKI and COL3A1 genes. Our aims are to validate the MPS approach using previous Sanger sequence data and to offer these genes in various Sanger sequencing panels as an interim strategy to reduce costs and turnaround times.

\section{2. 'IT IS A VERY LONELY PATH': EXPLORING EXPERIENCES OF ESTABLISHING A GENETIC SUPPORT GROUP IN VICTORIA}

Emma Swain', Margaret Sahhar', Laura Forrest ${ }^{2}$, Louisa Di Pietro ${ }^{3}$

\footnotetext{
${ }^{1}$ University of Melbourne, Melbourne, VIC, Australia

${ }^{2}$ Familial Cancer Centre, Peter MacCallum Cancer Centre, Melbourne, VIC, Australia ${ }^{3}$ Genetic Support Network of Victoria, Melbourne, VIC, Australia
}

The diagnosis of a genetic condition can be traumatic and life changing. Genetic support groups have an important role in providing mutual or peer support, advocacy, and assisting in public and professional education for families affected by a genetic condition. People may be motivated to start a support group for a variety of reasons, but this can be difficult, and there has been little prior research conducted on the process. This research, which was part of the Master of Genetic Counselling program at the University of Melbourne, aimed to explore how members of the Genetic Support Network of Victoria (GSNV) experienced establishing or attempting to establish a genetic support group in Victoria.

Seven semi-structured, in-depth interviews were conducted with nine participants. Using a narrative analytic approach, a number of concepts and themes were identified: participants found that setting up and running a support group could be lonely; they experienced being a support person for others as confronting; and felt they needed to acquire skills to help them establish their group effectively. Participants also needed mutual support and information from a genetic support group, and worked in partnership with health professionals and umbrella organizations to establish their group. These findings have implications for genetic counselors who have skills in providing emotional support, training, and facilitating the running of, and access to, support groups. This research also suggests a role for the 
GSNV in providing practical assistance to those who wish to start a genetic support group in Victoria.

\section{GIVING AND RECEIVING: COMPARING THE OPINIONS OF PARENTS, PEDIATRICIANS AND GENETIC HEALTH PROFESSIONALS ABOUT CHROMOSOMAL MICROARRAY RESULTS \\ Erin Turbitt $^{1,2}$, David Amor ${ }^{1,2}$, Jane Halliday ${ }^{1,2}$, Sylvia Metcalfe ${ }^{1,2}$ \\ ${ }^{1}$ Murdoch Childrens Research Institute, Melbourne, VIC, Australia \\ 2 University of Melbourne, Melbourne, VIC, Australia}

Background: Chromosomal microarray (CMA) testing for childhood investigations has been on the Australian Medical Benefits Schedule since 2010, enabling a range of clinicians to order the test. Despite CMAs offering improved diagnostic yields, they result in increased detection of results of uncertain clinical significance. Limited research has explored the opinions of parents, paediatricians and genetic health professionals regarding CMA result disclosure in a clinical setting. Our aim was to investigate and compare the perspectives of these three groups. Methods: A questionnaire was designed following 15 qualitative interviews with key informants and piloted with 11 parents. Victorian parents were recruited at community-based immunization sessions. Email invitations were sent to paediatricians and genetic health professionals via listservs. Results: To date, 121 parents, 95 paediatricians and 52 genetic health professionals responded. Interim analysis indicates all health professionals preferred to disclose all CMA results, regardless of level of certainty. At most, $75 \%$ of parents reported definitely wanting to receive all result types, with the remainder being unsure (KruskalWallis: $p<.001)$. Despite this widespread preference to disclose uncertain results, health professionals reported experiencing difficulty explaining them to parents. Parents perceived uncertain results as having less practical value (e.g., access to services/treatment) and predicted these results would cause more anxiety than a certain result. Conclusion: This study offers novel insight into the opinions of parents and health professionals about the disclosure of uncertain CMA results in Australia. Results demonstrate a degree of discordance between health professionals and consumers, which should be considered in development of optimal national guidelines for reporting genomic information.

\section{TEN YEARS OF MYOTONIC DYSTROPHY TESTING IN A DIAGNOSTIC LABORATORY - INTERESTING CASES AND OVERALL FINDINGS}

Evelyn Douglas', Rachael Catford', Jan Liebelt', Lesley McGregor ${ }^{2}$, Kathryn Friend $^{2}$

I SA Pathology, Adelaide, SA, Australia

2 SA Pathology - WCH, Adelaide, SA, Australia

There are two known loci for myotonic dystrophy, DM1 and DM2. Myotonic dystrophy type 1 (DM1) is an autosomal dominant disorder associated with expansion of a CTG trinucleotide repeat, in a non-coding region of the DMPK gene. There are mild, classic and congenital forms of DM1 with severity correlated with increased number of CTG repeats. Symptoms range from cataract and mild myotonia to muscle wasting and weakness, cardiac conduction abnormalities and severe generalized weakness at birth, with respiratory insufficiency and early death in the congenital form. We present a summary of molecular genetic analysis (a combination of PCR and Southern Blot analysis) of samples referred to the laboratory for myotonic dystrophy testing over the past 10 years and discuss interesting cases. These include a family ascertained by a female presenting very late in pregnancy with polyhydramnios. The fetus was found to carry a DMPK CTG repeat within the congenital range and the mother identified to carry a CTG repeat within the classic range. Another interesting case was where the partner of an individual known to carry an expanded CTG allele was also found to carry an allele with a CTG repeat within the mildly affected range, highlighting the importance of genetic counseling. We have recently introduced testing for DM2, another autosomal dominant disorder involving myotonia and muscle dysfunction. DM2 is caused by expansion of a complex repeat within intron 1 of the CNBP gene, and we are currently screening symptomatic individuals, previously negative for DM1. The summary of these findings will be presented and discussed.

\section{CASCADE SCREENING FOR ARGININOSUCCINIC ACIDURIA IN A FAMILY: ANOTHER BENEFIT OF NEWBORN SCREENING}

\section{Galit Tal}

Murdoch Childrens Research Institute, Melbourne, VIC, Australia

Argininosuccinic Aciduria (ASA) is due to a deficiency in Argininosuccinate Lyase (EC 4.3.2.1) enzyme and is the second most common urea cycle disorder. The age of onset and clinical presentation are varied. It can present in the neonatal period or even adulthood. Clinically, it presents with recurrent acute encephalopathy due to hyperammonaemia and chronic encephalopathy with neuro-cognitive deficits, behavioral abnormalities and learning disabilities. These manifestations have been attributed not just due to the harmful effects of hyperammonaemia, but also effects of elevated argininosuccinic acid, CNS creatine and NO deficiencies secondary to arginine deficiency.

Newborn screening (NBS) for ASA has been available in Victoria since 2002 as part of the expanded newborn screening program using Tandem Mass Spectrometry. Long-term follow-up of patients diagnosed by NBS has shown a favorable but variable outcome.

A newborn baby born to consanguineous parents was diagnosed with ASA following a positive newborn screening result. Baby was asymptomatic. Family history, however, revealed that her two older brothers (6 and 4 years of age), who were born overseas have significant protein aversion and neuro-developmental concerns Cascade screening confirmed that they were also affected. Treatment was initiated in all 3 children. Improvement in concentration and behavior was observed in the affected boys.

Our family highlights the importance of cascade screening in families whose children were born before the expanded NBS era or in countries with limited NBS programme. It also highlights the significance of a dietary assessment in a child with undiagnosed neuro-developmental concerns.

\section{CRYPTIC FAMILIAL TRANSLOCATION PREDISPOSING TO ADJ1 AND ADJ2 SEGREGANTS IN LIVE BORNS}

George Davis, Tina Lillis-Peters, Rachel Beddows

Wellington Regional Genetics Laboratory, Wellington, New Zealand

We report a familial cryptic translocation $[\mathrm{t}(13 ; 22)(\mathrm{q} 12 ; \mathrm{q} 11.2)]$ where two separate unbalanced segregants have been observed in live births (Adj1 \& Adj2). Due to the breakpoints, and chromosomes involved, it was not possible to distinguish between the balanced and unbalanced segregants using cytogenetic analysis alone and in situ hybridization studies were required to determine the nature of the rearrangements present in family members. Both unbalanced segregants gave rise to well characterized syndromes: DiGeorge and Microduplication 22q11.1. The implications of carrying cryptic acrocentric translocations is discussed. 
27. POLYMORPHISM OF TUMOR NECROSIS FACTOR-ALPHA -238 IS ASSOCIATED WITH NON ALCOHOLIC STEATOHEPATITIS; A STUDY IN INDONESIAN POPULATION

Hery Djagat Purnomo ${ }^{1,2}$, Farmaditya EP Mundhofir ${ }^{2}$, Kasno Kasno ${ }^{1,2}$, Edi Sudjianto $^{1,2}$, Hirlan Hirlan ${ }^{1,2}$, Darmono Darmono ${ }^{1,2}$, Daldiyono Daldiyono ${ }^{3}$, Robert Djokomoeljanto ${ }^{1,2}$, Sultana MH Faradz ${ }^{2}$

${ }^{1}$ Dr Kariadi Hospital, Semarang, Central Java, Indonesia

${ }^{2}$ Faculty of Medicine Diponegoro University, Semarang, Central Java, Indonesia

${ }^{3}$ Dr.Cipto Mangunkusumo Hospital, Jakarta, Indonesia

Background: Non-alcoholic steatohepatitis (NASH) is a subset spectrum of NAFLD that can progress toward cirrhosis. Tumor necrosis factor-alpha (TNF-alpha) polymorphism play a significant role in the regulation of immune cells, liver injury and inflammation. Furthermore, it is associated with the incidence and development of Non Alcoholic Fatty Liver Disease (NAFLD). In this study, the association between TNF-alpha (-238 and -308) polymorphism) and the development of NAFLD was evaluated. Methods: A total of 155 subjects (80 NAFLD cases and 75 controls) were included. Liver biopsy was performed in all NAFLD cases. Plasma TNF-alpha was measured in all subjects. Polymorphisme of TNF-alpha promoter gene -308 and -238 were identified using PCR-RFLP confirmed with direct sequencing. Results: Liver biopsy established the diagnosis of NASH in 29 cases. There was no association between the incidence of NAFLD with TNF-alpha polymorphism at the TNFalpha -308 or the $-238(p>.05)$. The prevalence ratio of TNF-alpha polymorphism -238 was significantly higher for subject with NASH $(p<.02)$. On the contrary, there was no different prevalence ratio for TNF-alpha polymorphism -308 ( $p>.05)$. To our surprise, novel polymorphism of TNF-alpha -216 was revealed in a subject with possible NASH, high plasma TNF-alpha level $(20.27 \mathrm{pg} / \mathrm{cc})$ and very high value of Homeostasis Model of Assessment - Insulin Resistance HOMA-IR (22.73). Conclusion: Polymorphism TNFalpha -238 is likely to be a risk factor for NASH in Indonesia. The identification of new possible polymorphism of TNF-alpha -216 requires further study in more samples.

\section{INVESTIGATION OF GRIEF AND LOSS CHALLENGES EXPERIENCED BY AUSTRALASIAN GENETIC COUNSELORS}

Holly Wojcicki ${ }^{1}$, Linda Humphreys' ${ }^{1}$, Jane Fleming ${ }^{1}$, Aideen McInerney ${ }^{2}$

${ }^{1}$ Griffith University, Brisbane, QLD, Australia

${ }^{2}$ University of Queensland Diamantina Institute, Brisbane, QLD, Australia

To date, the comfort of Australasian genetic counselors with client grief and loss has been largely unexplored. We surveyed members of the Australasian Society of Genetic Counsellors (ASGC) in order to assess their self-reported comfort with grief and loss. Additionally, we considered the impact of specific factors such as years of experience, comfort with uncertainty and perceived adequacy of training.

Participation was voluntary, with 65 of the 139 ASGC genetic counselors completing the survey. Fifty-seven $(87.7 \%)$ respondents reported feeling comfortable with client grief and loss, while 52 $(80 \%)$ respondents felt that they were adequately trained to deal with client grief and loss. Approximately two thirds of genetic counselors (40/65) also reported having experienced an influential personal loss. Bivariate logistic regression analysis demonstrated both years of clinical experience $(p=.02)$ and comfort with uncertainty $(p=.02)$ to be positively associated with comfort around grief and loss. However, neither of these factors were found to be significant predictors of comfort with client grief and loss in multivariate analysis. Subsequent bivariate analysis of these variables found years of experience and comfort with uncertainty to be positively correlated $(p=0.02)$. Similarly, a positive association was established between comfort with uncertainty and self-care practice.
This study has provided some insight into the unique experiences of Australasian genetic counselors with client grief and loss. It is believed that these findings can inform genetic counseling training and practice.

\section{THE AUSTRALIAN GENETICS CONSUMER: FIRST EMPIRICAL DATA OF AUSTRALIAN CONSUMERS' KNOWLEDGE AND EXPECTATION OF DIRECT-TO-CONSUMER PERSONAL GENOME TESTING}

Jacqueline Savard, Julie Mooney-Somers, Ainsley Newson, lan Kerridge

Centre for Values, Ethics and the Law in Medicine, Sydney, NSW, Australia

Direct-to-consumer personal genome testing (DTC-PGT) includes for-profit genetic testing services available via the Internet. They offer consumers probability assessments for physical traits, disease conditions and/or disease susceptibilities and information about ancestors including their continental origins, possible ethnicity and any links they might have with celebrated historical figures. Despite being available in Australia since 2007, little is known about the public's understandings and perceptions of DTC-PGT. We report a study designed to address this gap.

Between November 2011 and April 2012, 270 adults completed a short online survey about general knowledge of DTC-PGT, understandings of what information DTC-PGT provides, perceived value of such information and intentions to pursue this type of testing.

Results suggest Australian consumers have limited awareness of DTC-PGT. Only 40\% identified DTC-PGT as available in Australia, with a further $53 \%$ unsure, and only $43 \%$ believed it to be legal. This low awareness may in part explain the low intention to take up DTC-PGT among the sample; only $27 \%$ indicated such an interest. Interestingly, when the perceived value of genetic information provided by these tests is compared with known health histories and family ancestral stories, the majority of respondents reported they believed genetic information to be as important as other forms of self-knowledge. While few participants expressed an interest in pursuing DTC-PGT, the perceived utility of these tests provides further evidence of public support for genetic explanations of health and illness and suggests that there may be a large potential market for DTC-PGT.

30. FETAL DNA CONCENTRATIONS IN CONVERTED DNA EXTRACTED FROM MATERNAL PLASMA CAN BE INCREASED USING RESTRICTION ENZYME (RE) DIGESTION Jang Jih Chen', Kek Heng Chua', Elizabeth George ${ }^{2}$, Jin Ai Mary Anne Tan'

${ }^{1}$ University of Malaya, Kuala Lumpur, Malaysia
${ }^{2}$ University Putra Malaysia, Kuala Lumpur, Malaysia

Introduction: Current prenatal diagnosis for beta-thalassemia involves invasive procedures. Circulating fetal DNA in maternal plasma has been used for non-invasive prenatal diagnosis (NIPD) in certain genetic disorders. Epigenetic studies revealed that specific CpG sites in the beta-globin gene are less methylated in fetal DNA compared with maternal DNA. This differentiation in methylation status may be used as an approach for NIPD for beta-thalassemia provided sufficient concentrations of fetal DNA are available. Methods: Extracted free-circulating DNA (fcDNA) in maternal plasma was subjected to bisulfite conversion. Two informative $\mathrm{CpG}$ sites located close to the beta-mutation at codon $41 / 42$ were previously identified using converted maternal and CV DNA. Maternal plasma was obtained from pregnant mothers with the beta-mutation at codon 41/42. Amplification was performed on the converted fcDNA (cfcDNA), followed by double RE digestions to digest sequences that contain the methylated cytosines. The undigested DNA was used in real-time PCR (RT-PCR) to quantitate the frequency of the normal 
and mutant alleles to deduce the foetal genotype. Results: Four cfcDNA were successfully amplified. Normal allele frequency was higher in two c-fcDNA, lower in one c-fcDNA and the same in one c-fcDNA compared to the mutant allele frequency. This indicates the fetuses to be normal, mutant and carrier respectively. The genotype of the foetuses using RE digestion was in concordance with results from the conventional Amplification Refractory Mutation System. Conclusions: In this preliminary research, prenatal diagnosis using double RE digestions in conjunction with RT-PCR quantitation may allow determination of fetal genotype for non-invasive prenatal diagnosis.

\section{A CASE OF MILLER SYNDROME WITH LIVER DISEASE AND PARADOXICAL BIOCHEMICAL FINDINGS}

Jason Pinner' ${ }^{\text {, Kevin Carpenter }}{ }^{2}$, Keith Ooi ${ }^{3}$, Michael Bamshad ${ }^{4}$

${ }^{1}$ Royal Prince Alfred Hospital, Sydney, NSW, Australia

${ }^{2}$ Children's Hospital at Westmead, Sydney, NSW, Australia

${ }^{3}$ Sydney Children's Hospital, Sydney, NSW, Australia

${ }^{4}$ University of Washington, Seattle, WA, USA

Miller syndrome (Genee-Wiedemann syndrome, postaxial acrofacial dysostosis POADS (OMIM\#263750) is a multiple congenital anomaly syndrome with normal intellect caused by partial deficiency of dihydrorotate dehydrogenase (DHODH), an enzyme in the pyrimidine biosynthetic pathway. Our patient had characteristic features of Miller syndrome (cleft palate, congenital heart disease, oligodactyly and dysmorphic features) and was born prematurely at 27/40 complicated by RDS, heart failure and neonatal cholestasis. Identification of heterozygous frameshift and missense mutations in DHODH provided molecular confirmation.

His neonatal cholestasis was extensively investigated but no other etiology was found. Intraoperative cholangiogram and liver histopathology excluded biliary atresia. Liver biopsy revealed unusual generalized feathery degeneration of hepatocytes, mild fibrosis and cholestasis. Immunohistochemical staining for familial cholestatic diseases was negative. His conjugated hyperbilirubinaemia resolved at 6 old on ursodeoxycholic acid but liver biochemistry remains abnormal with progressive hepatomegaly. Growth continues below the third centile. Neurodevelopment is normal.

Urine metabolic screen identified paradoxically elevated orotate and uracil, and very low level of dihydroorotate (normally not detected) but no other abnormalities. Spontaneous conversion of dihydroorotate to orotate was excluded. Plasma amino acids were normal. Similar pyrimidine pathway biochemical findings have recently been reported in two molecularly confirmed cases of Miller syndrome 2 but not found in others. However, hepatic involvement has not been previously reported. The urinary orotate may be a marker for patients at risk of hepatic disease. We think the biochemical abnormalities and hepatic features are secondary to the underlying metabolic defect through an unknown mechanism. One possibility is an unidentified pathway bypassing DHODH.

\section{2. 'I DON'T HAVE A NAME FOR IT TODAY': EXPLORING COMMUNICATION IN PEDIATRIC CLINICAL GENETIC CONSULTATIONS}

Jean Paul $^{1,2}$, Sylvia Metcalfe ${ }^{1,2}$, Lesley Stirling ${ }^{2}$, Jan Hodgson ${ }^{1,2}$

${ }^{1}$ Murdoch Childrens Research Institute, Melbourne, VIC, Australia

${ }^{2}$ The University of Melbourne, Melbourne, VIC, Australia

Background: A large proportion of clinical geneticists' workload includes investigations for children with developmental delay where the underlying cause often remains unknown. New diagnostic tech- nologies provide hope for a diagnosis for many of these children. However, results generated through use of these technologies increases the complexity of communication and uncertainty during genetic consultations. To date there has been limited research into the process of paediatric genetic consultations. This project investigates the process and experiences for clinicians and parents during these consultations. Methods: This qualitative project investigated consultations across four Australian states. Theoretical framework: Symbolic Interactionism meaning is derived, created and modified through social interactions. Data included: audio-recorded consultations $(n=32)$, parent pre-consultation surveys $(n=32)$, and parent $(n=32)$ and clinician $(n=11)$ post-consultation interviews. Detailed microanalysis (content, thematic and discourse) was completed across data sets for enhanced understanding, triangulation and analytical rigour. Results: Overall, the content of the consultations was similar although clinicians appeared to have different 'styles' of communicating and interacting with families. Parents largely understood the information and had realistic expectations regarding diagnoses. Where expectations were not met, parents were often disappointed. Clinicians described many professional challenges working in this area, both practical and emotional. In particular, they described the frustration of frequently being unable to answer parents' questions regarding the cause of the child's delay. Conclusion: Detailed analysis of three complementary data sources has provided rigorous and unique perspectives on impacts of new genetic technologies for clinicians and parents. Findings from this study will inform best practice in this area of medical communication.

\section{3. 'CHROMOSOMES ARE LIKE RECIPE BOOKS': HOW CLINICIANS EXPLAIN NEW TECHNOLOGIES IN THE CLINIC}

Jean Paul $^{1,2}$, Sylvia Metcalfe ${ }^{1,2}$, Lesley Stirling ${ }^{2}$, Jan Hodgson ${ }^{1,2}$

${ }^{1}$ Murdoch Childrens Research Institute, Melbourne, VIC, Australia

${ }^{2}$ The University of Melbourne, Melbourne, VIC, Australia

Background: A core role of the clinical geneticist is to explain complex and abstract genetics at an appropriate level in order to facilitate parents' understanding of their child's condition. The introduction of new diagnostic technologies provides hope for a diagnosis for many children with developmental delay. However, results generated through use of these technologies increases the complexity and uncertainty during genetic consultations. We explore ways in which clinicians explain new genetic technologies and compare results with clinicians' and parents' perspectives. Methods: This qualitative project investigated pediatric genetic consultations across 4 Australian states. Theoretical framework: Symbolic Interactionism - meaning is derived, created and modified through social interactions. Data included: audio-recorded consultations $(n=32)$, parent pre-consultation surveys $(n=32)$, and parent $(n=32)$ and clinician $(n=11)$ post-consultation interviews. Detailed microanalysis (content, thematic and discourse) of clinicians explaining genetic results was compared with parent and clinician interviews. Results: Clinicians used a variety of approaches when explaining new genetic technologies and genetic test results. Some prepared pictures and diagrams to explain chromosomes and genes, contrasting new technologies to what had been possible in the past. Other clinicians used analogies such as making a cake and following recipes to explain the effect of gene changes. Parents appeared to mostly understand the clinicians' explanations. More successful explanations were those where parents felt comfortable asking questions and felt clinicians had listened to their concerns. Conclusion: This research demonstrates the importance of adapting communication strategies to suit parent understanding. Findings from this study will inform best practice in this area of medical communication. 


\section{A COMPLEX PRENATAL REFERRAL SHOWING AN ABNORMAL AMNIOCENTESIS RESULT WITH DISCORDANCE FROM THE INITIAL ABNORMAL DIRECT CVS RESULT}

Joanne Wardrop', Andrew Beddow', Rachel Beddow', Kim Flintoff', Kate Gibson $^{2}$

${ }^{l}$ Wellington Regional Genetics Laboratory, CCDHB, Wellington Hospital, Wellington, New Zealand

${ }^{2}$ Genetic Health Service NZ, New Zealand

We present a case report of a complex abnormal prenatal referral, tested by both CVS and amniocentesis. The patient was 40 years old and initially had prenatal testing overseas for advanced maternal age and a family history of Down syndrome. The initial CVS report indicated a mosaic trisomy 13 result from direct CVS analysis. There was no long-term CVS result from the overseas lab 4 weeks after sampling.

Upon return to New Zealand the patient elected to have a followup amniocentesis. Rapid aneuploidy testing of the amniocentesis sample (using both QF-PCR and interphase FISH) showed no evidence of the trisomy 13 detected on the previous direct CVS. The initial trisomy 13 result is therefore likely due to confined placental mosaicism (CPM). Karyotype analysis from the amniocentesis revealed a mosaic result with two abnormal cell lines and a normal male cell line. Further investigation, including FISH, M-FISH and $\mathrm{aCGH}$, identified that the major cell line contained an additional small derivative chromosome 9. The second abnormal cell line contained the additional derivative chromosome 9 along with an additional derivative chromosome 15 .

This case demonstrates the complexity of some prenatal referrals with differing CVS and amniocentesis results. It also shows the value of traditional FISH based techniques in conjunction with aCGH for delineation of some cytogenetic abnormalities.

\section{DETECTION OF WILMS TUMOUR GENE 1 MUTATIONS IN ACUTE MYELOID LEUKEMIA}

Joëlle Marivel ${ }^{1,2}$, Anabel Kearney ${ }^{1,2}$, Lindsay Dunlop ${ }^{2}$, Penelope Motum ${ }^{2}$, Samantha Day ${ }^{2}$, Anne-Marie Watson ${ }^{2}$, Danny Hsu ${ }^{2}$, Nicholas Viiala ${ }^{2}$, Micheal Harvey $^{2}$, Adam Bryant ${ }^{2}$, Lye Lin $\mathrm{Ho}^{2}$, David Rosenfeld ${ }^{2}$, Silvia Ling ${ }^{2}$

${ }^{1}$ Ingham Institute for Applied Medical Research, Sydney, NSW, Australia
${ }_{2}^{2}$ Department of Haematology, Liverpool Hospital, Sydney, NSW, Australia

The Wilm's Tumour gene 1 (WT1) encodes a zinc-finger transcription factor protein which consists of a transcriptional regulatory domain (exons 1 to 6 ) and $4 \mathrm{C}$-terminal zinc-finger domains (exons 7 to 10). Mutations in the WT1 gene were identified nearly 20 years ago in acute myeloid leukemia (AML) patients and have since been reported at a frequency of $10 \%$ in cytogenetically normal $(\mathrm{CN})$ AML. The mutated WT1 AMLs have been shown to be associated with failure to achieve complete remission, low survival rates and possibly drug resistance. WT1 mutations are independently a poor prognosis factor but have also been reported in association with other gene mutations such as FLT3-ITD and NPM1. Therefore, detecting WT1 mutations in CN-AML patients becomes an important part of patient management. To investigate the prevalence and clinical implications of the WT1 gene mutations in the Sydney South West Health District, we retrospectively screened for the presence of mutations in exons 7 and 9 of the WT1 gene in 46 patients who presented at Liverpool Hospital between 2009 and 2012. Our cohort was composed of $26 \mathrm{CN}-\mathrm{AML}, 11$ cytogenetically abnormal(CA) AML and 9 non-AML. Using capillary electrophoresis and bi-directional sequencing, we found mutations in exon 7 in 2 of the 46 patients. Both of these patients were cytogenetically normal and had relapsed refractory disease. These are the first reported WT1 mutations in AML in Australia. Future work would include a larger cohort as well as gene expression studies to monitor the levels of WT1 as the disease progresses.

36. DEVELOPMENT OF THE NEW ZEALAND RARE DISEASE BIOBANK

John Forman, Olivia Baddeley

NZ Organisation for Rare Disorders, Wellington, New Zealand

The New Zealand Rare Disease Biobank was initiated after discussion of the concept at the NZ Organisation for Rare Disorders conference in 2004. The biobank initially developed a virtual collection of naturally occurring animal models of rare disease, then applied for ethical approval to collect human samples, receiving this in 2010. A sample management database was developed, facilities and consumables acquired. It can collect samples, as well as providing an option for samples that would be prone to being 'lost', that is, at retirement of a researcher. It has obtained a few samples, but has operated at a very low capacity, due to limited resources to actively promote or facilitate major collection.

NZIRDR Ltd, the charitable research arm of NZORD, is working in partnership with the University of Otago Pathology Department to develop the biobank, to encourage, promote and facilitate rare disease research. It aims to approach donation at support group level, ideally with the assistance of a clinical or research affiliate, to assist coordination of donation and increase numbers of donations of similar disease types. Subject to resource limitations and collaboration opportunities, NZIRDR is preparing to increase collection and thus opportunities for rare disease research.

Developing the biobank was quite a learning process, and challenges continue. These include resource constraints, practical aspects of consent processes across various ages, and managing consent where different people and locations are involved. Opportunities exist for clinicians and researchers interested in biobanking and research to work with us to develop this resource.

\section{PRE-IMPLANTATION GENETIC DIAGNOSIS SEVEN YEAR'S EXPERIENCE IN NEW ZEALAND.}

\section{John Forman}

NZ Organisation for Rare Disorders, Wellington, New Zealand

Guidelines which authorized the use of PGD in New Zealand were issued in May 2005 and 6 months later the Minister of Health announced public funding to ensure equitable access to this procedure, by those at risk of inherited genetic diseases in future pregnancies. The first procedures commenced around the middle of 2006 once clinics had set up their equipment and procedures and organized the required testing facilities. This presentation provides figures on the number of procedures, the range of conditions which triggered the procedure, and outcomes in live births, over the past 7 years. Discussion includes aspects of administration of PGD funding through District Health Boards, regional access issues, and the monitoring of outcomes by ACART, the Advisory Committee on Assisted Reproductive Technologies.

\section{RARE DISEASE PATIENT REGISTRY - THE TIME IS NOW} John Forman, Olivia Baddeley

NZ Organisation for Rare Disorders, Wellington, New Zealand

There is a shift of focus among international rare diseases researchers and funders. An ambitious goal of finding diagnostics for most rare diseases, and therapies for 200 of them by 2020 , 
has been set by the International Rare Disease Research Consortium (IRDiRC). The need for large, global, shared rare disease data is seen as a vital part of this goal, involving formation and connection of patient registries throughout the world, with anonymized data shared in a common information pool accessible to researchers.

We envisage for the NZ part of the puzzle and the NZ rare disease community a secure web-based patient centered registry that allows for online registration, consent, and inputting of medical information by individuals. The vision is to have a NZ registry for all rare diseases ( $\sim$ approximately 7,000$)$ with multiple disease subsets in it, managed in NZ by NZIRDR Ltd, the research arm of the NZ Organisation for Rare Disorders.

The collective de-identified data of the registry would allow interoperability and data sharing with the global rare disease databases, to optimize use of the data. Information technology is now at a point where the vision of global registry data is a possibility, with the advent of such advances as cloud computing and high speed internet. Global registry efforts that now link over 40 different neuromuscular registries, demonstrate the validity of this approach. The technology is here and the collective thinking and global vision for rare diseases is clear - the future is here.

\section{GENETIC CONTROL FOR GENE EXPRESSION AND ITS} IMPACT ON DISEASE SUSCEPTIBILITY

Joseph Powell', Grant Montgomery ${ }^{2}$, Peter Visscher ${ }^{3}$

\footnotetext{
${ }^{1}$ University of Queensland, Brisbane, QLD, Australia

2 Queensland Institute of Medical Research, Brisbane, QLD, Australia

${ }^{3}$ University of Queensland Diamantina Institute, Brisbane, QLD, Australia
}

Gene expression levels have been extensively studied in humans as a mechanism that can underlie complex phenotypes such as common disease. It is well established that variation in expression levels can be a contributing factor in the susceptibility to disease; placing greater importance on understanding the sources of variation for gene expression. Gene expression levels are known to be heritable and many studies have been extremely successful at identifying genetic loci that influence expression levels (eQTL). Significant SNPs from genome-wide association studies (GWAS) are highly enriched for eQTL and recent ENCODE work has revealed most lie in regulatory hotspots.

We have completed a series of studies focusing on multiple aspects of genetic control underlying gene expression. Here we summarize these studies and present our methods showing the integration of these results to identify expression effects on disease. In brief, using data collected as part of the Brisbane Systems Genetics Study (BSGS) we have: (1) Mapped eQTL with both additive and non-additive effects; (2) Identified loci influencing variance heterogeneity for gene expression; (3) Shown differences in the genetic control for gene expression between tissues; (4) Decomposed sources of variation in gene expression; (5) Identified epistatic effects for gene expression; (6) Revealed genetic covariance between expression and disease.

We have shown that sources of variation for expression can differ between genes and between tissues, leading to a multifactorial impact on disease system-wide. Gene expression data and the results we present are highly dimensional. Therefore, we have developed a set of simple web-based tools to visualize and understand the results across the genome.

\section{ALPHA CARDIAC ACTIN MUTATION CO-SEGREGATES WITH HYPERTROPHIC AND DILATED CARDIOMYOPATHY AS WELL AS BEING ASSOCIATED WITH CONDUCTION DISEASE}

Julie McGaughran' ${ }^{\text {, Lauren Hunt }}{ }^{\text {, Louise McCormack }}{ }^{2}$, Michael Christiansen ${ }^{3}$, John Atherton ${ }^{2}$

\footnotetext{
${ }^{1}$ Genetic Health Queensland, Brisbane, QLD, Australia

${ }^{2}$ Department of Cardiology, RBWH, Brisbane, QLD, Australia

${ }^{3}$ Statens Serum Institut, Copenhagen, Denmark
}

Mutations in alpha cardiac actin (ACTC1) have been rarely reported in nonsyndromic familial dilated cardiomyopathy (DCM) and hypertrophic cardiomyopathy (HCM). We report a family with both $\mathrm{HCM}$ and DCM associated with conduction disease requiring pacing that co-segregates with an ACTC1 missense mutation.

A 40-year-old woman with HCM presented for genetic testing. Her father was also said to have HCM and died while awaiting cardiac transplantation. She was aware that two of her paternal uncles were also said to have cardiomyopathy. An ACTC1 missense mutation (c.76 G>A) was identified on genetic screening. Further family studies were suggested to clarify the pathogenicity of the change. One uncle initially presented at the age of 51 years with atrial fibrillation associated with normal LV size, wall thickness and systolic function. He subsequently developed symptomatic atrioventricular (AV) conduction disease, dilated cardiomyopathy and clinical heart failure at 64 years of age and pacing was performed in view of 3rddegree AV block (despite no negative chronotropic agents). LMNA mutation screening was negative. He also had the ACTC1 missense mutation. Another paternal uncle diagnosed with $\mathrm{HCM}$ also had the ACTC1 missense mutation.

Conclusion: This case illustrates the phenotypic variation associated with sarcomere mutations. ACTC1 mutations have been previously reported in a small number of HCM and DCM families, however, has not been previously reported in associated with conduction disease.

\section{IDENTIFICATION OF NOVEL RNA SPLICING MUTATIONS IN FAMILIAL CANCER GENES - EXPERIENCE IN A DIAGNOSTIC LABORATORY}

\section{Karen Carpenter, Jinyi Qin, Caitlin Edwards, Edward Edkins}

PathWest, Perth, WA, Australia

With the increasingly widespread use of high throughput sequencing, many sequence variants of unknown pathological significance are being generated. A significant proportion of these could affect RNA splicing. The best-known splicing mutations are those that affect the canonical splice sites at intron/exon boundaries, However, missense or even translationally silent variants within exons can cause defects in splicing by activating cryptic splice sites or disrupting regulatory elements such as exonic splicing enhancers (ESEs). In addition, sequence changes deep within introns can also have an effect. These mutations can result in complete exon skipping, deletion of part of the exon or inclusion of intronic sequence.

The consequence of a sequence variant on splicing in most routine diagnostic laboratories can usually only be determined using splicing analysis software. However, the reliability of these programs is difficult to assess. Patient RNA analysis is often not performed in the majoritory of diagnostic laboratories due to difficulties in both methodology and the need to obtain patient RNA from appropriate tissues. In addition, nonsense mediated mRNA decay may also mean some mutations escape identification. In this laboratory we have developed a routine cDNA sequencing approach using RNA from patient PHA-stimulated lymphocytes. Using this technique we have identified a number of novel mutations causing defects in 
splicing in a range of cancer predisposition genes including MLH1, NF1, PMS2, SDHD and ATM.

\section{DETECTION OF MUTATIONS IN JAK2 V617F NEGATIVE MYELOPROLIFERATIVE DISORDERS}

Karen Ambler, Musei Ho, Madelyn Zawitkowski, Wendy Parker, Glenice Cheetham

\section{SA Pathology, Adelaide, SA, Australia}

JAK2 V617F is an acquired mutation found in myeloproliferative disorders (MPD) such as polycythemia vera (PV), essential thrombocythemia (ET) and myelofibrosis (MF). Detection of JAK2 V617F assists in diagnosis however it does not distinguish between the disorders. There are additional mutations which can aid the diagnosis of MPD in particular, JAK2 exon 12 mutations which appear to be restricted to cases of PV; and MPL mutations which are more likely to be found in ET and MF.

Our laboratory has validated a JAK2 V617F test using Sequenom's iPLEX chemistry and MALDI-TOF MS. This technique is easily amenable to multiplexing and our single well assay was designed to test for both the JAK2 V617F and the MPL W515L mutations. We present here the validation of the MPL W515L test. The incidence of the W515L mutation in undiagnosed MPD patients is $1 \%$ compared to a reported $6 \%$ in patients with diagnosed ET or MF.

The most common JAK2 exon 12 mutation is the N542-E543 deletion and currently we test for these mutations using sanger sequencing. In our laboratory the incidence of JAK2 exon 12 mutations in $\mathrm{V} 617 \mathrm{~F}$ negative patients is $0.5 \%$, compared to a reported incidence of $3 \%$ in patients with JAK2 V617F negative PV. Our custom designed panel for MPD mutations has been demonstrated to be a reliable, sensitive, high throughput test for the detection of JAK2 V617F and MPL W515L. Future modifications of this assay may include the addition of JAK2 exon 12 mutations and other MPL mutations.

\section{DUCHENNE/BECKER MUSCULAR DYSTROPHY - INCIDENTAL FINDINGS ON ARRAY CGH}

Kathy Cox, Linda Burrows, Evelyn Douglas, Jillian Nicholl, Wendy Waters, Christopher Barnett, Christopher Manning, Sui Yu, Kathryn Friend

SA Pathology - Women's and Children's Hospital, Adelaide, SA, Australia

Duchenne Muscular Dystrophy (DMD) and Becker Muscular Dystrophy (BMD) are X-linked recessive muscle disorders caused by mutations within the dystrophin gene. Array CGH detects copy number variation and is routinely requested in patients referred for intellectual disability, behavioral issues and autism. Since the implementation of Array CGH in South Australia (August 2010), five patients with deletions or duplications involving the dystrophin gene have been identified. MLPA analysis for clarification of the copy number change within the dystrophin gene was performed and, where possible, cascade screening of at risk relatives was undertaken after appropriate counseling. Of the incidental findings, three female carriers were identified; cascade screening identified several other carrier females, but fortunately no males affected with D/BMD were identified in these families. Two males with copy number changes in the dystrophin gene were also identified. The first case was from a foetus with bilateral renal agenesis (autopsy sample). An in-frame duplication of exons 17-27 of the dystrophin gene was identified - to date no family members have presented for screening for this mutation. The second case was a 17 year old male referred for intellectual disability, aggression and behavioral problems. An in-frame deletion of exons 44-45 of the dystrophin gene was identified. Interestingly this patient was also identified to have a duplication of 6q27 involving TBP and PDCD2. Clinical significance will be discussed. This work highlights the importance of following up incidental findings such that cascade screening for clinically important disorders, unrelated to the initial referral, can be instigated.

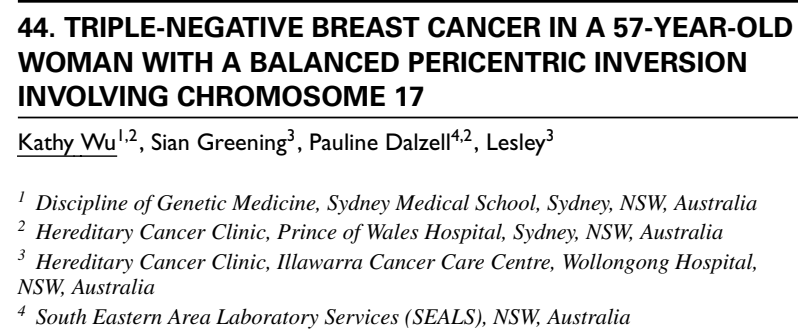
WOMAN WITH A BALANCED PERICENTRIC INVERSION INVOLVING CHROMOSOME 17

$\underline{\text { Kathy Wu }}^{1,2}$, Sian Greening ${ }^{3}$, Pauline Dalzell ${ }^{4,2}$, Lesley ${ }^{3}$

\footnotetext{
${ }^{\prime}$ Discipline of Genetic Medicine, Sydney Medical School, Sydney, NSW, Australia

${ }^{2}$ Hereditary Cancer Clinic, Prince of Wales Hospital, Sydney, NSW, Australia

${ }^{3}$ Hereditary Cancer Clinic, Illawarra Cancer Care Centre, Wollongong Hospital, NSW, Australia

${ }^{4}$ South Eastern Area Laboratory Services (SEALS), NSW, Australia
}

Background: Triple-negative (estrogen receptor/progesterone receptor/Her-2 negative) breast cancers are known to be associated with germline BRCA1 mutations. The proband, SG, developed a triple-negative invasive breast cancer at age 57 years, with no known family history of breast/epithelial ovarian cancer. She has a child with Klinefelter syndrome (XXY), another with trisomy 21 , and the third child was identified prenatally to have an apparently balanced pericentric inversion involving chromosome 17, 46,XX,inv(17)(p13.1q21.31), and subsequently developed a granulosa cell ovarian tumour at age 7.5 years. SG was confirmed to be a carrier of this inversion. Objectives: To investigate the possibility of disruption of gene(s) relevant to oncogenesis in close vicinity of breakpoints of a balanced pericentric inversion, $\operatorname{inv}(17)(p 13.1>17 q 21.31)$, in SG, as an underlying cause of her cancer, and potential implications to family. Methods: Molecular and cyto-genetic investigations, including BRCA1 and BRCA2 sequencing and multiple-ligation probe amplification (MLPA), 400k array comparative genomic hybridization $(\mathrm{aCGH})$, fluorescent insitu hybridization (FISH) analyses for BRCA1 (17q21.31) and p53 (17p13.1). Results: Sequencing and MLPA of BRCA1/2 in SG did not identify any mutation. FISH analysis showed that BRCA1 locus was not disrupted. Other investigations are underway. Conclusion: The possibility of disruption of long-range BRCA1 regulatory elements on the inverted chromosome is not excluded. Further studies are awaited to see whether other gene(s) at/near the breakpoints are disrupted to contribute to the tumor phenotype. Interestingly, a case of granulosa cell ovarian tumour has been reported in association with germline p 53 mutation. Mechanism of different chromosomal aneuploidies arising from the pericentric inversion was also hypothesized.

\section{TWO NOVEL MOUSE MODELS OF HUMAN CRANIOFACIAL DYSMORPHOLOGY}

\section{Kerry Miller', Tiong $\operatorname{Tan}^{2}$, Megan Welfare', Peter Farlie}

${ }^{1}$ Murdoch Childrens Research Institute, Melbourne, VIC, Australia
${ }^{2}$ Victorian Clinical Genetics Services, Melbourne, VIC, Australia

Birth defects are structural or biochemical abnormalities that are present at birth, and are the leading cause of infant morbidity and mortality, affecting $3-5 \%$ of live births. Approximately one third of all birth defects involve the craniofacial structures, where they are frequently associated with other clinical characteristics such as defects in the limbs and/or other organ systems. To underpin our studies on the genetic control of morphogenesis and congenital malformation in humans we undertook a comprehensive 
recessive mutagenesis screen to identify novel mouse models of human dysmorphology. We have identified novel ENU mouse models of two distinct human craniofacial dysmorphologies. Kanyon embryos have a mid-facial cleft, ocular anomolies (micropthalmia) and a variable mid-brain exencephaly, phenotypes that mimic human frontonasal dysplasia. The facial cleft often varies in severity, from a disastrous lesion completely disrupting the face to a discrete cleft lip and palate phenotype. Thus kanyon, in its mildest form, may also be a model for cleft lip and palate, the most common and well known craniofacial abnormality in humans. Mutant snoopy embryos display a unilateral facial hypoplasia phenotype that involves the mandible, mid-face and ears. These characteristics are very similar in appearance to the human condition Goldenhar syndrome. We are analyzing the exomes of both the snoopy mouse and a number of familial Goldenhar patients in order to identify the developmental origins of Goldenhar syndrome and the first genetic cause of this disorder.

\section{DOUBLE TROUBLE: A CASE OF TWO BALANCED ROBERTSONIAN TRANSLOCATIONS IN ONE COUPLE}

\author{
Kirsty Mann' , JA Taylor, Susan Fawcett ${ }^{2,}$ \\ ${ }^{\prime}$ Royal Melbourne Hospital, Melbourne, VIC, Australia \\ 2 Victorian Clinical Genetics Services, Melbourne, VIC, Australia
}

We describe a rare case in which two different balanced Robertsonian translocations were detected in a couple planning their first pregnancy. The woman was the first found to have a balanced translocation; her karyotype revealed she is a carrier of rob(14q21q). Testing had been prompted by her reportedly identical twin sister having a daughter with Down syndrome resulting from an unbalanced form of the translocation.

During follow-up genetic counseling her partner revealed a family history of an uncle who also had Down syndrome and so a karyotype was requested for him. The result found him to also be a carrier of a balanced Robertsonian translocation involving chromosomes 14 and 15 .

We present the implications of these balanced translocations for the couple's first pregnancy, the outcome of prenatal diagnosis, and the genetic counseling issues faced throughout the process. We describe the possible conceptions to be considered and the testing strategies undertaken to analyse the risks of Down syndrome and other genetic conditions that could potentially arise from uniparental disomy of the imprinted chromosomes 14 and 15.

\section{A DIAGNOSIS OF PITT-HOPKINS SYNDROME IN A 21 MONTH OLD CHILD AND DETECTION OF MATERNAL SOMATIC MOSAICISM AT 18021.2}

Kit Doudney', Vivienne Bickley', Monique Robertson', Jane Watt', Mac Gardner², Jill Taylor', Alexa Kidd', Peter George'

${ }^{1}$ Canterbury Health Laboratories, Christchurch, New Zealand

${ }^{2}$ Genetic Health Service, New Zealand

Pitt-Hopkins syndrome (PTHS) is a neurodevelopmental disorder often caused by heterozygous mutations in TCF4, a transcription factor important during embryonic development. Typical PTHS characteristics are severe intellectual disability, breathing abnormalities and abnormal facial morphology including a wide mouth. Following a diagnosis of global developmental delay at 21 months of age, patient 1 was referred to our laboratory for arrayCGH. We detected an approximately $600 \mathrm{~kb}$ heterozygous deletion at $18 \mathrm{q} 12.2$, encompassing the 5' half of TCF4 and $300 \mathrm{~kb}$ of upstream genomic sequence.
Analysis of the proband's phenotypically normal parents revealed a maternal mosaic pattern of deletion at this locus, identified by FISH in $11 \%$ of interphase nuclei and confirmed in metaphase cells. Mosaicism at 18q21.2 (TCF4) is rare, with only three examples reported. This finding provides evidence that TCF4 haploinsufficiency underlies this child's developmental disorder, that it is maternally inherited, and provides the basis for screening of future pregnancies with the possibility of prenatal genetic diagnosis for the family concerned.

\section{HEPARIN SULFATE PROTEOGLYCANS (HSPGS) AND RUNX2 MEDIATE HUMAN BREAST CANCER EPITHELIAL CELL PROLIFERATION}

Larisa Haupt $^{\prime}$, Rachel Okolicsyani ${ }^{1}$, Andre van Wijnen ${ }^{2}$, Simon $\mathrm{Cool}^{3}$, Lyn Griffiths

${ }^{1}$ Griffith University, Brisbane, QLD, Australia

${ }^{2}$ Mayo Clinic, USA

${ }^{3}$ Institute of Medical Biology, Singapore

Heparan sulfate proteoglycans (HSPGs) are key components of the extracellular matrix where they mediate cell proliferation, invasion and cellular signaling. Previous studies have observed roles for HSPG core proteins including syndecans (SDC1-4) and glypicans (GPC1-6) in breast cancer and hepatocellular carcinoma. In this study we examined the expression profile of HSPGs core proteins along with their key initiation and modification enzymes in human breast cancer epithelial cells. Gene expression in relation to cell proliferation was examined in two human breast cancer cell lines (MCF-7 and MDA-MB-231 cells) following treatment with the HS agonist heparin. The addition of heparin increased gene expression of chain initiation and modification enzymes including EXT1 (MCF-7; $p=0.0002$, D1 inc; MDA $p=1.77 \times 1 \mathrm{e}-8$, D3 inc) and NDST1 (MCF-7 $p=0.016$, D1 inc; MDA $p=3.31 \times 1 \mathrm{e}-5$, D3 inc). In addition significant changes in core protein gene expression was observed including SDC1 (MCF-7 $p=0.02$, D1 inc; MDA $p=$ $0.011, \mathrm{D} 3 \mathrm{dec}$ ) and GPC6 (MCF-7 $p=1.6 \times 1 \mathrm{e}-7$, D3 inc; MDA $p$ $=2.94 \times 1 \mathrm{e}-9, \mathrm{D} 3 \mathrm{inc})$. When we investigated the HS/Runx2 axis in MDA-MB-231 cells, we observed reduced cell migration following Runx 2 siRNA down regulation. Finally we investigated the cultures for associated expression changes in the Wnt pathway. We observed increased proliferation of the more invasive MDA-MB-231 cells associated with activation of members of the Wnt signaling pathway. Specifically, there was substantial up-regulation of the AXIN1, 9 fold inc, $p=1.4 \times 1 \mathrm{e}-5$, WNT4A, 15 fold inc, $p=0.006$ and MYC, 6 fold inc, $p=0.0003$ genes at D5 in MDA-MB-231 cells. In contrast, MCF-7 cells showed a small increase in Wnt genes at D1 (AXIN1, 1.5 fold inc, $p=0.01$; MYC, 1.3 fold inc, $p=0.005$; FZD5, 1.9 fold inc, $p=0.007$ ). The significant gene expression changes observed within HSPG initiation and modification enzymes, core proteins, and key partners in Wnt signaling support the theory that HSPGs are critically involved in cellular proliferation and differentiation in a cancer model. In addition, as in other cell model systems, there appears to be a central role for Runx2 in HS-mediated cellular events.

\section{HOW MUCH PROTEIN IS IN THAT? AN INVESTIGATION} OF PROTEIN COUNTING APPLICATIONS

Leah Vandervliet

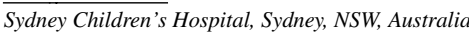

Protein-restricted diets are an essential part of the treatment of a variety of inborn errors of protein metabolism. Dietitians utilize 
a variety of education resources. New technologies such as applications ('apps') containing nutritional data could become part of the dietitian's toolkit. The purpose of this study was to identify available nutrition-based apps and compare their nutritional data.

Six applications were included in the study: Easy Diet Diary, CalorieKingTM, MyFitnessPal, PKU Calc, DietWellTM and Muscle Gainer-Protein Tracker. Common foods containing moderate and high amounts of protein were selected for analysis $(n=38)$.

Apps varied in their dietary data, cost, and additional features. Protein values for the selected foods were assessed in each application and compared to values listed in the ASIEM Low Protein Handbook. The most common reason for foods being excluded from analysis $(n=48)$ was their absence from the database $(n=$ $24,50 \%)$. The serving size $(\mathrm{g})$, protein per serve $(\mathrm{g})$ and weight of food containing $1 \mathrm{~g}$ protein $(\mathrm{g})$ for each food was compared using a paired $t$ test. There was no significant difference in the mean serving size $(\mathrm{g})$ or mean protein per serve $(\mathrm{g})$ between the apps. There was a significant difference for the mean weight of food containing $1 \mathrm{~g}$ protein in PKU Calc (30.2g, SD 21.1, $\mathrm{p}=0.03)$ and Muscle Gainer Protein Tracker (30.3g, $S D$ 34.9, $p=.0482$ ). Individual differences in protein values for foods was not statistically significant.

Nutrition-based applications have the potential to enhance management, but have their limitations. Differences in protein values may be clinically significant, thus it is recommended they be used as part of comprehensive dietetic education. of these (95\%) accessing within 4 years of testing, and all selecting individual reporting.

\section{POPULATION-BASED CARRIER SCREENING FOR CYSTIC FIBROSIS: A SYSTEMATIC REVIEW OF 23 YEARS OF RESEARCH}

Liane loannou', Belinda McClaren', John Massie ${ }^{2}$, Sharon Lewis ', Sylvia

Metcalfe' ${ }^{\prime}$, Laura Forrest' ${ }^{\prime}$, Martin Delatycki'

${ }^{1}$ Murdoch Childrens Research Institute, Melbourne, VIC, Australia

${ }^{2}$ Royal Children's Hospital, Melbourne, VIC, Australia

Cystic fibrosis (CF) is the most common, severe, autosomal recessive disease with a prevalence of 1 in 2,500-3,500 live births and a carrier frequency of 1 in 25 among Northern Europeans. CF population-based carrier screening has been possible since CFTR, the disease-causing gene, was identified in 1989. This review provides a systematic evaluation of the literature on population-based $\mathrm{CF}$ carrier screening from the last 23 years focussing on: uptake of testing; how to offer screening; attitudes, opinions and knowledge; factors influencing decision-making; and follow-up after screening. Recommendations are given for the implementation and evaluation of future carrier screening programs.

\section{EVALUATION OF DEFERRED SELECTION OF RESULT REPORTING STRATEGIES IN COMMUNITY GENETIC SCREENING FOR AUTOSOMAL RECESSIVE DISORDERS}

\author{
Leslie Burnett $^{1,2,3}$, Ann L Proos ${ }^{1,2}$, Kristine Barlow-Stewart ${ }^{1,3,4}$, Harry \\ Aizenberg ${ }^{1,5}$ \\ ${ }^{1}$ Australasian Community Genetics Program, Sydney, NSW, Australia \\ 2 PaLMS Pathology North, Sydney, NSW, Australia \\ ${ }^{3}$ University of Sydney, Sydney, NSW, Australia \\ ${ }^{4}$ NSW Health - Centre Genetics Education, Sydney, NSW, Australia \\ ${ }^{5}$ Wolper Jewish Hospital, Sydney, NSW, Australia
}

Community genetic screening for autosomal recessive (AR) disorders such as Tay-Sachs disease (TSD) or cystic fibrosis (CF) is a highly effective way of achieving reduced disease incidence and restoration of reproductive confidence. Two broad screening strategies are relevant: (1) Individual client (after testing, results are issued to the client); and (2) Anonymous couple-based (results are not issued to the clients; instead, the [prospective] couple are advised if the union is 'at-risk' of an AR-affected child for the screened condition). Some religious communities insist on anonymous couple screening, especially when arranged marriages are being contemplated. Clients electing individual screening generally disqualify themselves from eligibility for later enrolment in anonymous couple screening.

To avoid premature decision-making on reporting strategy, and facilitate opportunistic uptake, we developed a 'Deferred' program: clients could access screening, but did not receive $*$ any $*$ result at time of testing. Instead, they could contact the laboratory at any future time to request entry into the individual or the couple-based programs.

We describe the outcome from a 3-year period (1997-1999) of 2,298 NSW high school students (age 16-19) participating in organized TSD/CF community screening offering a Deferred program. While Deferred result reporting increased client choice and ethical practice and resulted in increased uptake, the utility of the resultreporting strategy was inferior to standard strategies. Even after 15 years, only $33 \%$ of students had accessed results, with the majority
52. EXPERIENCES OF COUPLES BOTH IDENTIFIED AS CARRIERS OF CYSTIC FIBROSIS IDENTIFIED THROUGH A POPULATION-BASED CARRIER SCREENING PROGRAM

Liane loannou' ', Martin Delatycki', John Massie ${ }^{2}$, Jan Hodgson', Sharon Lewis'

${ }^{1}$ Murdoch Childrens Research Institute, Melbourne, VIC, Australia

${ }^{2}$ Royal Children's Hospital, Melbourne, VIC, Australia

Background/Aims: Cystic fibrosis (CF) is the most common severe, autosomal recessive disease among Caucasians. A population-based $\mathrm{CF}$ carrier screening program was implemented in Victoria, Australia in 2006. The aim of this study was explore the experiences of couples that were both identified as carriers of CF through this program. Methods: Between January 2006 and December 2010, 10 carrier couples were identified. All couples were invited to participate in this qualitative study by a genetic counselor associated with the program. Interviews were semi-structured and analyzed using inductive content analysis. Results: A total of nine interviews were conducted, seven couple interviews and two individual interviews. The main reasons for having screening were high perceived severity of CF, high perceived susceptibility and to avoid having a child with CF. All couples experienced shock on learning their carrier couple result. Six of the nine couples were pregnant at the time of screening and all had prenatal diagnosis. Two of the pregnancies were affected with participants reporting grief upon receiving the prenatal diagnosis result. Both couples elected termination of the affected fetus. Three of the nine couples had no further children since being identified as a carrier couple. Of the remaining six couples, four utilized prenatal diagnosis and two utilized preimplantation genetic diagnosis for subsequent pregnancies. All participants informed at least one family member of their carrier status. Conclusions: Couples were unprepared for a positive carrier couple result. However, all the couples changed their reproductive behavior as a result of their carrier status and informed family members of their increased risk. 


\section{PRIMARY ERYTHROMELALGIA: CASE REPORT,} MANAGEMENT, AND GENETIC COUNSELING

\author{
Lisa Ewans, lan Andrews, Anne Turner \\ ${ }^{l}$ Sydney Children's Hospital Network, Sydney, NSW, Australia
}

Primary erythromelalgia is a rare congenital disorder of neuropathic pain, due to mutations in the SCN9A gene. SCN9A encodes a voltage-gated sodium channel, Nav1.7, found mainly in nociceptive neurons, whose expression is regulated by inflammatory mediators. Symptoms are often refractory to treatment. We describe a case of a 6 year-old girl with intermittent burning pain, warmth, and erythema in her limbs, particularly affecting her palms and soles. Alleviation of symptoms by itching and running cold water over affected regions caused excoriated wounds. Impact of this disorder on the family was substantial, with a number of counseling issues. Identification of a de novo activating heterozygous SCN9A gene mutation confirmed clinical diagnosis. Management was multifaceted, involving treatment of skin lesions, introduction of medication targeted at alleviating pain, and specific interventions for the family. This case highlights the impact primary erythromelalgia can have, and the complexity of management in this disorder.

\section{ANALYSIS OF EYE DISORDER PHENOTYPES IN THE NORFOLK ISLAND GENETIC ISOLATE}

Lyn Griffiths', David Eccles ${ }^{2}$, Rod Lea ${ }^{2,3}, \mathrm{~A} \mathrm{Hewitt}^{3}$, Miles Benton ${ }^{2}$, Elizabeth Matovinovic ${ }^{2}$, Matthew Johnson ${ }^{4}$, Claire Bellis ${ }^{4}$, Joanne Curran ${ }^{4}$, J Blanjero ${ }^{4}$, D Mackey ${ }^{3}$

${ }^{l}$ Griffith University, Brisbane, QLD, Australia

${ }^{2}$ Genomics Research Centre, Griffith Health Institute, Gold Coast, QLD, Australia

${ }^{3}$ Centre for Eye Research, Melbourne, VIC, Australia

${ }^{4}$ Texas Biomedical Research Institute, San Antonio, TX, USA

Glaucoma and myopia are complex eye disorders affecting a large proportion of the population. The genetic determinants of these conditions and their underlying endophenotypes are only partially known. In this study we have utilized the Norfolk Island genetic isolate to investigate a number of ocular phenotypes. This population originates from a small number of British and Polynesian founders who are descendents of the Bounty mutiny, with the majority of the population forming a very large multigenerational pedigree. The large pedigree structure, founder effect and admixture of the Norfolk Island isolate offer advantages for studying the genetics of ocular phenotypes.

We statistically analyzed 25 ocular phenotypes measured in 801 individuals from Norfolk Island. We first performed principal components analysis (PCA) to account for intercorrelation among phenotypes. Focusing in on the founder core pedigree $(n=362)$, we then performed heritability screening of all univariate and multivariate traits to estimate $\mathrm{H} 2$, which was followed by pedigree-based GWAS to search for major susceptibility loci.

For the individual ocular phenotypes, significant $\mathrm{H} 2$ values ranged from 0.20 to 0.78 , with the highest $\mathrm{H} 2$ obtained for central corneal thickness. PCA revealed 9 factors that explained $82 \%$ of the total variance, with the top 4 factors being significantly heritable $(\mathrm{H} 2 \mathrm{~min}=0.58, \mathrm{P}<.05)$. Pedigree-based GWAS of all heritable traits revealed multiple SNPs that exceeded the empirical genomewide significance threshold $(\mathrm{P}<1.84 \sqrt{ }$ ó10-7). The results of this analysis highlight some potentially important loci underlying ocular phenotypes.
55. THE IMPACT OF PARTICIPATION IN GENETIC RESEARCH FOR FAMILIES WITH CLEFT LIP WITH OR WITHOUT CLEFT PALATE

Lynley Donoghue ', Laura Forrest ${ }^{2}$, Sahhar Margaret ${ }^{3}$, Supriya Raj ${ }^{4}$, Nicky

Kilpatrick $^{4}$

${ }^{1}$ Port Macquarie Genetics Service, Port Macquarie, NSW, Australia

${ }^{2}$ Peter MacCallum Cancer Centre, Melbourne, VIC, Australia

${ }^{3}$ Victorian Clinical Genetics Services, Melbourne, VIC, Australia

${ }^{4}$ Murdoch Childrens Research Institute, Melbourne, VIC, Australia

Very little is understood about the genetic contributions to isolated clefts of the lip with or without palate (CL/P). OzCleft, a familybased study currently underway at the Murdoch Childrens Research Institute in Victoria Australia, is examining the genotype:phenotype correlation. Families' participation involves clinical investigations of the child with CL/P, their unaffected sibling(s) and parents. Informal feedback from individuals involved in OzCleft suggested that participation had benefits for families. Therefore, this research aims to explore families' experiences of participating in the OzCleft study.

This study used a qualitative approach to investigate the impact of the OzCleft study on families, who included parents and their children. Semi-structured in-depth interviews were conducted with nine parents who had participated in OzCleft. Interviews were audio-taped, transcribed verbatim and analysed using thematic analysis.

All parents described participation as a positive experience for themselves and their families, and perceived benefits included an appreciation of the CL/P treatment experience by unaffected family members. Being involved in a genetic study raised issues for parents regarding the cause of CL/P. While some parents found the existence of a genetic component reassuring, in most cases it raised questions about the potential implications for future generations. Parents were largely unsure about how to communicate this information to their children and the value of this information.

This study highlights a possible lack of genetic understanding and perceived value of genetic information. Furthermore, communication of genetic information was lacking, highlighting the need for support from genetic health professionals in this area.

\section{USING SKYPETM AS AN OPTION FOR FACE-TO-FACE COUNSELING: OUR EXPERIENCE AT SYDNEY ULTRASOUND FOR WOMEN (SUFW)}

Lynn Hulston, Kristi Murphy

Sydney Ultrasound for Women, Sydney, NSW, Australia

Objective: To examine the use of video counseling as an effective means of consulting women compared to face-to-face counseling. Second, to demonstrate that those women who were given a high risk combined first trimester screening result (cFTS) and those where a fetal abnormality was identified received the same level of care and attention. Methods: Women attending SUFW for cFTS have a consultation following the ultrasound to discuss their results with a genetic counselor or a doctor. A prospective study was conducted using the SkypeTM system as an alternative method of consulting when a doctor or genetic counselor was not present at the site. Results: There were technology issues that caused difficulties in some instances, but the majority were easily resolved. Experience has allowed a better screening process for those whom a face-to-face consultation would be the better option and thus a video consult is not offered. An effective system for consulting women with a high risk cFTS result and women where a fetal abnormality has been identified has been developed which allows maximum care to be 
taken of these cases. Conclusion: SkypeTM counseling has been in use at SUFW since September 2012. Patients have been in favour of SkypeTM in preference to phone consultation as they know they are dealing with a 'real' person providing the same quality of care as they would experience in a face-to-face consultation.

\section{ERYTHROMELALGIA: A RARE GENETIC PAIN} SYNDROME

Mac Gardner', Dean Kilfoyle ${ }^{2}$

${ }^{1}$ Genetic Health Service, New Zealand

${ }^{2}$ Auckland City Hospital, Auckland, New Zealand

Erythromelalgia is a syndrome of pain and redness especially of the distal limbs, and in some cases is due to mutation in the voltage-gated sodium channel gene SCN9A. This gene is particularly expressed in the dorsal root ganglia of the sensory nerve pathway. The condition typically appears before adult age, but later onset is recorded. We have seen a woman presenting in her 30s with otherwise classic symptomatology, and in whom a 5' SCN9A mutation was identified. One other case from the literature with a mutation in the same codon was also associated with later onset. A slightly lesser effect upon dorsal root ganglion electrophysiology may be the basis of the attenuated development of disease due to 5 ' mutation.

58. CRIMINALITY AND CHROMOSOMES: WILL MICROARRAY CAST NEW LIGHT?

Rachel Beddow', Susanna Every-Palmer², Mac Gardner $^{3}$

${ }^{1}$ Cytogenetics Laboratory, Wellington Hospital, Wellington, New Zealand

${ }^{2}$ Regional Forensic Service, Kenepuru Hospital, Wellington, New Zealand

${ }^{3}$ Genetic Health Service, New Zealand

We describe the case of a 38-year-old man detained in a forensic psychiatric facility, in whom a diagnosis of a psychosis had been made. He had committed numerous crimes since teenage, against property and against person. He had come from a high-achieving, responsible family. His physical appearance was essentially unremarkable. We identified a de novo $\sim 1.6 \mathrm{Mb}$ deletion at $19 \mathrm{p} 13$, removing 9 known disease-causing (OMIM) genes; and we conclude that this deletion and his behavioral phenotype are related as cause and effect. We suggest that the subtler level of imbalance that microarray can achieve may make a case for testing in a forensic setting, and especially in cases where the psychological/behavioral phenotype is considerably incongruent with the family background.

\section{NEWBORN SCREENING FOR HYPEROXALURIA TYPE 3: A PROOF OF PRINCIPLE STUDY}

Maggie Tan', Nicholas Tzanakos', Frank Willis ${ }^{2}$, Nicholas Tzanakos ${ }^{1,3}$, Yaacov Frishberg ${ }^{4}$, James Pitt ${ }^{1,4}$

1 Victorian Clinical Genetics Services, Melbourne, VIC, Australia

2 Nephrology Department, Princess Margaret Hospital for Children, Perth, WA, Australia

${ }^{3}$ Murdoch Childrens Research Institute, Melbourne, VIC, Australia

${ }^{4}$ Division of Pediatric Nephrology, Shaare Zedek Medical Center, Jerusalem, Israel

Background: Primary hyperoxaluria type 3 ( $\mathrm{PH} 3$ ) is a childhood kidney stone disease caused by mutations in the HOGA1 gene which encodes for 4-hydroxy-2-oxoglutarate aldolase in the 4-hydroxyproline catabolic pathway. We recently identified a metabolic shunting mechanism that accounts for the production of oxalate from 4-hydroxy-2-oxoglutarate in $\mathrm{PH} 3$ and discovered increased urine levels of 4-hydroxy-2-oxoglutarate, 2,4dihydroxyglutaric and 4-hydroxyglutamic (4HGlu). The current study examines the feasibility of newborn screening (NBS) for PH3. Methods: 4HGlu levels were measured in a dried blood spot sample from a $\mathrm{PH} 3$ patient stored for two years using a simple modification of the current tandem mass spectrometry NBS method and compared to storage matched and fresh controls. Samples from premature babies (30-35 weeks) were also analysed to check for secondary 4HGlu increases due to increased 4-hydroxyproline metabolism. Results: The 4HGlu level was markedly increased in the stored PH3 sample $(37 \mu \mathrm{mol} / \mathrm{L})$ compared to storage matched $(<2 \mu \mathrm{mol} / \mathrm{L}, n$ $=40)$ and fresh controls $(<1.6 \mu \mathrm{mol} / \mathrm{L}, n=40)$. 4HGlu level $\mathrm{s}$ in premature babies $(n=20)$ were not significantly increased. Conclusions: These results demonstrate the potential feasibility of NBS for $\mathrm{PH} 3$. Further studies in larger cohorts of controls and $\mathrm{PH} 3$ patients are required to better define the screening metrics of $4 \mathrm{HGlu}$. While PH3 does not meet traditional criteria for a stand-alone NBS test, these results show that screening can be implemented as a simple 'add on' to the current panel of NBS disorders detected by tandem mass spectrometry. If $4 \mathrm{HGlu}$ has a high positive predictive value for PH3 then early detection of this disorder would be a useful addition to NBS.

\section{S-SULPHOCYSTEINE CAN BE INCREASED IN BACTERIAL OVERGROWTH SYNDROMES}

Mary Eggington, Kai Mun Hong, Ava Mishra, James Pitt

Victorian Clinical Genetics Services, Murdoch Childrens Research Institute, Melbourne, VIC, Australia

Background: Urinary S-sulphocysteine (SSC) is widely regarded as a sensitive and specific marker for inborn errors of metabolism affecting sulphite metabolism, that is, sulphite oxidase deficiency and defects affecting the biosynthesis of molybdenum co-factor. These defects often present in the neonatal period with intractable seizures and rapid screening is frequently required. We have been testing all urine samples sent to our laboratory for metabolic screening for SSC using tandem mass spectrometry. Several patients with intestinal bacterial overgrowth syndromes were found to have moderately increased SSC levels. Methods: Over a 1-year period, SSC urine levels from patients in whom bacterial overgrowth was known or suspected (HRBO group, $n=51$ samples from 34 patients) were compared with levels in patients not suspected to have bacterial overgrowth (LRBO group, $n=3691$ samples). Results: SSC levels were significantly higher $(p<.0003)$ in the HRBO group compared to the LRBO group with 15 samples (29\%) from the HRBO group having SSC levels above the 99 th percentile $(11 \mu \mathrm{mol} / \mathrm{mmol}$ creatinine) of the LRBO group. SSC levels in the HRBO group ranged up to $54 \mu \mathrm{mol} / \mathrm{mmol}$ creatinine while four sulphite oxidase or molybdenum cofactor deficiency patients ranged from 80 to 856 $\mu \mathrm{mol} / \mathrm{mmol}$ creatinine. Conclusions: Some patients with bacterial overgrowth can have increased urine SSC levels, presumably the consequence of overgrowth with sulphur metabolising bacteria and subsequent host conversion of sulphite to SSC. This effect is a potential confounder during screening for deficiencies of sulphite oxidase and molybdenum cofactor. 
61. CLINICAL FEATURES OF GORLIN SYNDROME. A CASE REVIEW SERIES

Michael Bogwitz, Brenda Greyling, Aamira Huq, Alison Trainer, Kirsty Mann, Jessica Taylor, Ingrid Winship

Royal Melbourne Hospital, Melbourne, VIC, Australia

Gorlin Syndrome (OMIM 601309), also known as Nevoid Basal Cell Carcinoma Syndrome (NBCCS), is a rare autosomal dominant condition hallmarked by the appearance of multiple basal cell carcinomas (BCCs), odontogenic keratocysts, syndromic facial features including macrocephaly as well as disorders of the skeletal, nervous, cardiac and various other systems. It is inherited via heterozygous mutations in the PTCH1 gene which upregulates the hedgehog signaling pathway. Many criteria have been proposed for the clinical diagnosis of Gorlin syndrome; however, none of these criteria have been proven to be particularly sensitive or specific in diagnosing this rare syndrome.

We present a case series of patients with a molecular and/or clinical diagnosis of Gorlin Syndrome since 2006. A total of eight families were seen, of which three were shown to have a pathogenic mutation in PTCH1. Two patients who met the clinical criteria did not have a mutation in PTCH1, while another did not undertake testing. Testing is in process for in a more recent patient, and in another the diagnosis is being further refined with the aid of imaging. Clinical features of these patients will be presented in further detail, including a man with 119 BCCs, a woman with her first BCC diagnosed at 10 years of age and a family with all affected family members being diagnosed with jaw cysts as teenagers. This pilot study will act as a platform for further investigation into the diagnosis and management of patients with Gorlin Syndrome in Australian clinical genetics services.

\section{WHOLE-GENOME SEQUENCING APPROACH TO THE DETECTION OF GENETIC RISK FACTORS FOR ISCHEMIC STROKE \\ Michael Black', WenZui Wang ${ }^{2}$, Youxin Wang ${ }^{3}$, Wei Wang'

\footnotetext{
${ }^{1}$ Edith Cowan University, Perth, WA, Australia

${ }^{2}$ Beijing Municipal Key Laboratory of Clinical Epidemiology, Beijing, China

${ }^{3}$ Capital Medical University, Beijing, China
}

Ischemic Stroke (IS) affects approximately 15 million persons worldwide and is the third commonest cause of death and a major cause of adult neurological disability. While risk factors like obesity, hypertension, smoking, physical inactivity or atrial fibrillation account for a significant proportion of the risk for stroke, genetic variation is also believed to contribute to the risk. While knowledge of the impact of environmental and modifiable risk factors well advanced, the identification of genetic variants that modify risk is only in its infancy, with Genome Wide Association Studies (GWAS) the method of choice. However, IS genetic architecture is complex, involving 5 disease subtypes (large-artery atherosclerosis, cardioembolism, small-vessel occlusion, stroke of other determined aetiology, and stroke of undetermined aetiology), and is likely to include rare or low frequency variants, including indels and structural variants, that cannot be detected with GWAS methodologies.

In this study we have performed whole-genome sequencing of individuals from a Chinese pedigree with a strong genetic predisposition to IS in order to explore the possibility of the existence such rare variants as genetic risk factors. From the whole-genome data of three cousins we have detected candidate rare indel variants as well as $>100$ candidate SNPs which have been confirmed in past GWAS projects as either directly associated with IS or associated with related conditions and physiological risk factors. It is concluded that these results support a cumulative pleiotrophic multivariant model for genetic risk in stroke. A whole-genome sequencing approach is recommended for future studies of stroke genetics.

\section{THE NZ NMD REGISTRY - LOWERING BARRIERS TO RESEARCH FOR GENETIC DISORDERS}

Miriam Rodrigues', Graeme Hammond-Tooke ${ }^{2}$, Rakesh Patel ${ }^{3}$, Donald Love', Alexa Kidd $^{4}$, Richard Roxburgh'

\footnotetext{
${ }^{1}$ Auckland City Hospital, Auckland, New Zealand

${ }^{2}$ Dunedin Hospital, Dunedin, New Zealand

${ }^{3}$ Starship Auckland City Hospital, Auckland, New Zealand

${ }^{4}$ Canterbury Health Laboratories, Christchurch, New Zealand
}

The NZ Neuromuscular Disease Registry (NZ NMD Registry) is part of TREAT NMD, an international network for the neuromuscular field that provides infrastructure to ensure the most promising new therapies reach patients as quickly as possible. Scientific advances over recent years have lead to substantial changes in the treatment of many neuromuscular diseases. New therapeutic strategies are being developed and, for some of these treatments, large studies involving patients from more than one country are required. Several new therapeutic strategies for neuromuscular diseases target specific genetic defects. When a clinical trial is being planned, it is very important that patients suitable for that trial can be found and contacted quickly. The best way of ensuring this can happen is to collect patients' details in a single database or 'registry' that contains all the information researchers will need, including each patient's particular genetic defect and condition-specific information likely to be relevant to clinical research.

The NZ NMD Registry enrols patients with any neuromuscular condition that is offered support by the Muscular Dystrophy Association of NZ - the majority of which are genetic in origin and include muscular dystrophies, inherited ataxias, spinal muscular atrophies and hereditary neuropathies but also include acquired conditions such as inflammatory myopathies and myasthenic syndromes. Of those diagnosed with a genetic condition only approximately half the NZ NMD registry participants have a genetic test result. We examine whether it is valid that genetic testing is pursued for reasons other than genetic counseling and predicting or confirming a clinical diagnosis.

\section{IDENTIFYING THE EDUCATION NEEDS OF HEALTH PROFESSIONALS UTILIZING READILY ACCESSIBLE GENETIC TESTING TECHNOLOGIES}

Mona Saleh', Gillian Shannon ${ }^{2}$, Kate Dunlop'

${ }^{1}$ Centre for Genetics Education, Sydney, NSW, Australia

${ }^{2}$ Bathurst Community Health Centre, Bathurst, NSW, Australia

Rapidly advancing technology and the consequent increasing availability of DNA screening and testing, means that genetics will impact on more medical interactions in the community than ever before. This has become apparent in recent years with the increasing availability and utility of chromosomal microarray (CMA) testing in individuals (usually children), with non-specific, unexplained intellectual disability or multiple congenital anomalies. CMA testing is available for use by non-genetics health professionals assessing patients and can detect small DNA copy number variants (CNVs) which would not have been identified using traditional chromosome karyotyping techniques.

Non-genetics health professionals have utilized genetic testing for many years; however, due to the nature of CMA testing, the likelihood that a result of unknown or unexpected significance for the 
patient is found has never been so high. There is currently no information available as to how CMA testing is being presented to individuals and families being offered testing, or whether non-genetics health professionals themselves are aware of the significance of all possible test outcomes. The Centre for Genetics Education (CGE) is currently undertaking a gap analysis in order to assess the education and resource needs of non-genetics health professionals with regards to CMA testing. The study design, challenges and findings of the analysis as well as recommended educational strategies will be presented.

\section{ASSESSMENT OF METHYLATION IN MISMATCH REPAIR GENES IN PATIENTS WITH COLORECTAL CANCER}

Musei Ho, Karen Ambler, Madelyn Zawitkowski, Glenice Cheetham

SA Pathology, Adelaide, SA, Australia

Differentiation of HPNCC from sporadic colorectal cancer (CRC) is important for patient management. Immunohistochemistry (IHC) of mismatch repair (MMR) genes is widely used to identify patients who may carry a germline MMR mutation, however epigenetic silencing by promoter methylation also results in abnormal IHC. We reviewed IHC, BRAF V600E and MMR methylation results for 34 patients to determine the usefulness of these tests. The BRAF V600E mutation has previously been identified as a surrogate marker for MLH1 promoter methylation in CRC. We compared the V600E mutation result with the detection of MLH1 promoter methylation by methylation-specific (MS)-MLPA (MRC Holland) in patients with abnormal MLH1/PMS2 IHC. The V600E mutation had a PPV of $86 \%$ for methylation. The NPV was $47 \%$ indicating that the absence of the V600E mutation does not confirm the absence of MLH1 methylation and testing by an alternate method may be warranted. IHC for MLH1/PMS2 had a PPV of $68 \%$ for MLH1 methylation but this combination of tests also identifies those patients with abnormal IHC without MLH1 methylation who are candidates for HNPCC germline mutation screening. Although promoter methylation of other MMR genes was detected in a small number of patients, the clinical consequences are uncertain and this testing should remain a research tool. IHC, BRAF V600E and MS-MLPA testing provides a useful diagnostic algorithm for stratifying patients with CRC. Using these tests in combination allows the differentiation of those patients carrying epigenetic alterations from those who may carry germline mutations.

\section{GENE VARIANTS WITHIN UCP2 AND UCP3 ARE ASSOCIATED WITH CHANGES IN ENDURANCE PERFORMANCE FOLLOWING HIGH-INTENSITY INTERVAL TRAINING}

Nir Eynon, Sarah Voisin, Mary Zhang, David Bishop

Victoria University, Melbourne, VIC, Australia

Individual differences in the responses to similar exercise training are a well-known occurrence, and are influenced by genetics. The mitochondrial uncoupling protein 2 (UCP2) Ala55Val (rs660339) and uncoupling protein 3 (UCP3) $-55 \mathrm{C}>\mathrm{T}$ (rs1800849) polymorphisms may explain some of this variability given that mitochondrial function has been associated with endurance performance. We have designed a High-Intensity Interval Training (HIIT) study, and used the bootstrapping method, to investigate the influence of these two polymorphisms on muscle performance.
Twenty-five moderately trained, unrelated Australian males (21.8 $\pm 0.7 \mathrm{y})$ underwent 4 weeks of closely supervised HIIT training. Peak power and the lactate threshold were measured during an incremental test, and endurance performance (20-km cycle time trial) was measured pre and post training. A linear model was developed for each polymorphism to estimate the effect of the genotypes on baseline physiological parameters and on their changes following HIIT (10,000 resamplings, significance level $=0.05)$.

Both polymorphisms were significantly associated with the training response. UCP2, Ala-carriers showed a greater increase in endurance performance, as marked by a greater decrease in time to complete the 20-km time trial, compared with Val-carriers (change in time trial, Val/Val: $3.02 \pm 6.9 \%$, Ala/Val: $-1.35 \pm 1.34 \%$, Ala/Ala: $5.3 \pm 1.32 \%$ ). UCP3, C/C homozygotes showed a greater increase in endurance performance compared with $\mathrm{T} / \mathrm{C}$ heterozygotes (change in time to complete the time trial, T/C: $-0.34 \pm 2.2 \%, \mathrm{C} / \mathrm{C}:-3.6$ $\pm 1.06 \%$ ). This is the first data suggesting a link between UCP2 Ala55Val and UCP3 -55C $>\mathrm{T}$ polymorphisms and increased endurance performance following high-intensity exercise training.

\section{MOSAICISM FOR AN UNBALANCED TRANSLOCATION - TWO RARE CASES}

Pauline Dalzell, Genevieve Temple, Alex Kovacic, Linda Lim, Shampa Bhattacharjee, Ana Messias, Toni Saville, Moh Ying Yip

SEALS Genetics, NSW, Australia

Autosomal unbalanced translocations in mosaic form are extremely rare. Here we present 2 cases referred to our laboratory over a 20year period. Our first patient was a 28 -year-old female who was referred for recurrent miscarriages. Two separate studies on peripheral blood revealed around $27 \% 46, \mathrm{XX}, \operatorname{der}(6) \mathrm{t}(6 ; 8)(\mathrm{q} 27 ; \mathrm{q} 22.1) / 46, \mathrm{XX}$ mosaicism and only $46, \mathrm{XX}$ cells in skin fibroblasts. This study was supported by FISH analysis and was unusual in that the patient manifested an unbalanced translocation though in mosaic form without any phenotypic effect. The second patient, a 2-year-old boy with global developmental delay and dysmorphism was initially ascertained using aCGH and confirmed with FISH/karyotyping. A $66 \%$ 46,XY,der(22)t(14;22)(q32.1;q13.3)/46,XY mosaicism was apparent on karyotype analysis. Monosomy for $22 \mathrm{q}$ involved the $22 \mathrm{q} 13$ deletion syndrome genomic region.

Possible mechanisms of formation of the two unbalanced rearrangements will be reviewed. With the increasing use of microarray technologies, the detection of unbalanced rare anomalies particularly involving small segments not easily detected on karyotype analysis would surely increase.

\section{CYSTIC FIBROSIS PREIMPLANTATION GENETIC DIAGNOSIS - IGNORING MENDEL'S FIRST LAW}

\section{Peter Field, Nicole Martin \\ Queensland Fertility Group Genetics, Brisbane, QLD, Australia}

Cystic fibrosis is the most common deleterious single gene disorder in those of northern European non-Hispanic ancestry. The HGSA recommend CFTR mutation screening prior to pregnancy if possible and that a couple should at least be made aware and offered CF screening with associated genetic counseling to make an informed decision. For those couples identified as carrier couples, there are reproductive options to help with not having affected babies. These options include prenatal testing, donor gametes (sperm and/or egg), adoption or Preimplantation Diagnosis (PGD). 
An IVF/PGD cycle does not guarantee a healthy pregnancy and in fact does not guarantee a pregnancy. Pre-IVF cycle counseling along with genetic counseling is a must before embarking on an IVF/PGD cycle. We present here data from couples whom underwent CF PGD and show why these embryos have been ignoring Mendel's First Law. In 10 consecutive patient couples undergoing CF PGD, the outcome was not the Mendelian expected. From 51 embryos tested, 10 were unaffected (no mutations detected) 20 were carriers (one of the parental mutations detected) and 21 were affected (two disease causing mutations detected in the embryo). This ratio of 1:2:2 has been consistent through many patients and is not consistent with the expected mendelian ratios. Patient expectations and pre-IVF/pre-PGD counseling may need to reflect these observations.

\section{FAMILIAL INHERITANCE OF A SUPERNUMERARY MARKER CHROMOSOME 22 WITH VARIABLE PHENOTYPIC EXPRESSION OF CAT-EYE SYNDROME}

\section{Putra Sutedja, Luke St Heaps, Artur Darmanian, Dale Wright}

The Children's Hospital at Westmead, Sydney, NSW, Australia

Structural rearrangement within the chromosome 22q11.2 region can cause a variety of genomic disorders. Cat-Eye Syndrome (CES) is due to partial trisomy/tetrasomy of the segment 22pter to 22q11.2, often involving a Supernumerary Marker Chromosome 22 (SMC 22). We present a familial SMC 22 with variable phenotypic expression. Cytogenetic analysis was performed on a newborn male with hearing loss, bilateral ear pits, anterior ear tags, bilateral hydronephrosis and hydroureter, and mild jaundice. The proband's mother is known to have hearing loss, with a family history of congenital hearing impairment.

Karyotyping of the proband showed an additional bi-satellited marker chromosome in 15 cells: 47,XY,+mar. FISH analysis using D14Z1/D22Z1 and RP11-701M12 probes identified the marker as being derived from chromosome 22 and consistent with a symmetrical type I CES chromosome containing 2 copies of CECR 1 gene. Microarray CGH studies further characterized the marker and showed triplication (4 copies) of the segment in the proband involving $\sim 1.1 \mathrm{Mb}$ segment within band 22q11.1q11.21 including 25 genes from POTEH to TUBA8.

Familial chromosome studies showed a mosaic form of the marker was also carried by the proband's mother and brother, with different phenotypic expression than in the proband. The mother is mildly affected, while the brother is apparently normal. The degree of mosaicism and variable penetrance of the amplified genes in the Cat-Eye Critical Region is likely to be responsible for the variation in the phenotype observed in this family.

\section{A CHILD WITH A SEVERE EARLY MANIFESTATION OF JUVENILE POLYPOSIS SYNDROME ASSOCIATED WITH A BMPR1A GENE MUTATION}

$\underline{\text { Rachel Susman', Marie-Pierre Buisine }{ }^{2} \text {, Looi Ee }}{ }^{3}$

${ }^{1}$ Genetic Health Queensland, Brisbane, QLD, Australia

${ }^{2}$ Molecular Oncology and Genetics, Lille University Hospital, Lille, France

${ }^{3}$ Department of Gastroenterology, Royal Children's Hospital, Brisbane, QLD, Australia

We report a 5-year-old girl who presented with a history of rectal bleeding and profound anemia. Subsequently a clinical diagnosis of Juvenile Polyposis Syndrome (JPS) was made, and genetic testing was undertaken. We discuss her clinical and molecular findings and review the literature on early onset JPS.

\section{CLINICAL GUIDELINE ADHERENCE AND PSYCHOSOCIAL WELLBEING IN INDIVIDUALS WITH HYPERTROPHIC CARDIOMYOPATHY AND THEIR AT-RISK RELATIVES}

Renee Johnson', Maria Constantinou', Belinda Gray ${ }^{2}$, Christopher Semsarian ${ }^{2}$, Jodie Ingles ${ }^{2}$

\author{
${ }^{1}$ University of Sydney, Sydney, NSW, Australia \\ ${ }^{2}$ Centenary Institute, Sydney, NSW, Australia \\ ${ }^{3}$ Department of Gastroenterology, Royal Children's Hospital, Brisbane, QLD, \\ Australia
}

Introduction: Hypertrophic cardiomyopathy (HCM) is an autosomal dominant condition characterized by left ventricular hypertrophy. Clinical management and surveillance of at-risk relatives is critical in preventing the most serious outcome of sudden cardiac death. This study sought to describe adherence to clinical management and surveillance guidelines. Methods: Service utilization data was obtained from HCM families enrolled in the Australian Genetic Heart Disease Registry. Clinical and genetic data were collected, and a subset approached to complete a cross-sectional survey (Hospital Anxiety and Depression Scale [HADS], Medical Outcomes Survey Short Form-36v2 [SF-36v2], and Adjustment and Worry about HCM scale). Results: 334 individuals were included (76\% with $\mathrm{HCM}$ and $24 \%$ at-risk; $52 \%$ males, mean age $48-19$ years). Of these, $84 \%$ had visited a specialized cardiac genetic clinic. A subgroup of 17 individuals completed a detailed survey and $12(71 \%)$ reported being adherent to clinical guidelines. There were trends for better adjustment and less worry about HCM, improved healthrated quality of life and better psychological wellbeing in those who adhere to management guidelines. This is an on-going study and recruitment is continuing. Conclusions: Our preliminary data indicate there may be differences in the psychosocial status of individuals who do not adhere to clinical management and surveillance guidelines. Improved knowledge of these differences will provide a basis for strategies to better target this group.

\section{IMPROVED DIAGNOSTIC YIELD IN XY DISORDERS OF SEXUAL DEVELOPMENT WITH NEXT-GENERATION SEQUENCING}

Richard King, Ivan McGown, James Harraway, David Cowley

Mater Pathology, Brisbane, QLD, Australia

The management of XY disorders of sexual development (DSDs) is challenging. Patients may present at birth with ambiguous genitalia or later in life with absent or delayed puberty, primary amenorrhea or virilization. The degree of sexual ambiguity is broad, with phenotypes ranging from complete sex reversal to the under-virilized male. Multiple genes are involved in sex determination and differentiation and phenotypic heterogeneity is common to variants in each gene. Biochemical testing in these subjects is complex, time consuming and often equivocal.

To date most genetic investigation has been limited to the androgen receptor (AR), but in approximately $50 \%$ of patients no pathological variant is found. We report results on a cohort of 36 samples from patients with XY DSDs who did not have detectable AR mutations. These samples were tested using an in-house multi-gene XY DSD panel, on an Illumina MiSeq (Illumina Inc, San Diego, CA) platform. Early identification of the etiology in XY DSDs may assist with decisions concerning sex-of-rearing in those patients 
presenting at birth and will be informative in estimating recurrence risks within families.
75. PRRT2: ONE GENE, ONE MUTATION, MANY PHENOTYPES

Sarah Heron' ${ }^{1}$, Yeh Sze Ong', Simone Yendle ${ }^{2}$, Jacinta McMahon ${ }^{2}$, Samuel

Berkovic $^{2}$, Ingrid Scheffer ${ }^{2}$, Leanne Dibbens'

${ }^{1}$ University of South Australia, Adelaide, SA, Australia

${ }^{2}$ University of Melbourne, Melbourne, VIC, Australia

PRRT2 codes for a protein hypothesized to play a role in presynaptic neurotransmitter release. Heterozygous mutations in PRRT2 are largely associated with three neurological disorders: the seizure disorder benign familial infantile epilepsy (BFIE), the movement disorder paroxysmal kinesigenic dyskinesia (PKD) and infantile convulsions and choreoathetosis syndrome (ICCA), in which BFIE and PKD occur in the same patient or family. Heterozygous PRRT2 mutations have also been found in patients with hemiplegic migraine and other paroxysmal movement disorders. Over 380 families with PRRT2 mutations have been described, with $80 \%$ of families having a recurrent frameshift mutation. There are also two reports of homozygous mutations in PRRT2 causing infantile seizures and intellectual disability (ID).

Two other genes associated with benign infantile seizure disorders, KCNQ2, and SCN2A, are also associated with infantile epileptic encephalopathies (IEEs), in which seizures and ID occur. We hypothesized that PRRT2 mutations may also cause IEEs. We tested 220 patients with IEEs for mutations in PRRT2. No mutations were seen in this patient cohort. Homozygous PRRT2 mutations are a rare cause of infantile seizures with ID and heterozygous PRRT2 mutations do not cause IEEs as well as benign infantile seizures, in contrast to KCNQ2 and SCN2A. Instead, a distinct spectrum of neurological phenotypes encompassing benign infantile seizures, paroxysmal movement disorders and migraine is associated with heterozygous PRRT2 mutations. This spectrum is likely to be related to the hypothesized role of the PRRT2 protein in neurotransmitter release, perturbation of which is expected to cause neurological disorders. search groups. Individuals who participate online accept website terms and conditions, including disclaimers, waivers and limitations in liability, which are specified by the companies. It is necessary to understand how these companies proceed in order to provide commentary that informs best practices and ethical standards. Therefore, the online policies and documents of the companies are examined for issues concerning informed consent, data privacy and risk management. Observations will be offered about the robustness of safeguards and privacy assurances for individuals choosing to participate in studies conducted by these companies.

\section{DISCREPANCIES BETWEEN QF-PCR PRENATAL SEX CHROMOSOME RESULTS AND FULL CULTURE RESULTS - THE IMPLICATIONS FOR GENETIC COUNSELORS}

\section{Sarah Long}

Genetic Services of Western Australia, Perth, WA, Australia

Prenatal QF-PCR results allow parents at increased risk of chromosome anomalies to receive preliminary results $24-48$ hours after a diagnostic procedure. Rapid aneuploidy testing is currently carried out for chromosomes 13, 18, 21 and the sex chromosomes and the risk of discrepant results between QF-PCR and full culture karyotyping is considered low, and is usually ascribed to mosaicism. While a rapid normal result can reassure parents, three recent cases observed in Western Australia have highlighted issues regarding discrepancies in sex chromosome anomalies. The cases will be outlined here and the genetic counseling implications will be explored in an attempt to prevent negative outcomes in future cases.

\section{NEXT GENERATION SEQUENCING: INVESTIGATING MITOCHONDRIAL VARIATION IN MIGRAINE SUSCEPTIBILITY THROUGH HIGH THROUGHPUT FULL MITOCHONDRIAL GENOME SEQUENCING}

Shani Stuart ${ }^{1}$, Miles Benton ${ }^{2}$, Bridget Maher $^{3}$, David Eccles ${ }^{3}$, Heidi Sutherland ${ }^{3}$, Richard Allcock ${ }^{4}$, Rod Lea ${ }^{3}$, Larisa Haupt ${ }^{3}$, Lyn Griffiths ${ }^{3}$

${ }^{1}$ Griffith University, Brisbane, QLD, Australia

${ }^{2}$ Genomics Research Centre, New Zealand

${ }^{3}$ Genomics Research Centre, Griffith Health Institute, Gold Coast, QLD, Australia

${ }^{4}$ University of Western Australia, Perth, WA, Australia

Migraine is a neurological disorder characterized by recurring and often unilateral, throbbing head pain. It affects approximately $12 \%$ of the population with affected individuals predominantly females, suggesting that $\mathrm{X}$ linked and/or mitochondrial inheritance may be involved. We hypothesise that migraine development is influenced by mitochondrial dysfunction and given the role of mitochondria in both neurological disorders and energy production, mitochondrial variants may play a role in the pathogenesis of this disease. Initially a pilot project was conducted to evaluate NGS technology in full mitochondrial genome sequencing and assess the utility of this technology for variant identification. A small selection of individuals $(n=48)$ from the genetic isolate Norfolk Island population were chosen and sequenced using the Ion Torrent platform. We then expanded the work to include an additional 270 samples from the core pedigree, providing full mitochondrial sequence information for the entire Norfolk Island cohort. Following sequencing, FASTQ 
sequences were aligned using SAMTOOLS. Each aligned FASTA sequence was then compared to the updated Cambridge reference genome to identify variants and haplogroups for each sample. Thus far a rare Polynesian haplogroup has been identified along with other variants with known disease associations. Statistical modeling will be used to examine the association of mtDNA variants and haplogroups with heritable migraine traits in addition to modeling the effects of key environmental factors on mtDNA and ancestry associations. Future work will investigate gene expression changes in key metabolic pathway genes to further examine association between nuclear encoded variants affecting mitochondrial function and migraine susceptibility.

\section{CONFIRMATION OF A HIGH FREQUENCY OF THE 118 KB FILIPINO DELETION IN SARAWAK BETA-THALASSAEMIA PATIENTS USING HIGH RESOLUTION MELTING ANALYSIS \\ Siew Leng Kho', Kek Heng Chua', Saw Sian Chin², Gaik Bee Ong ${ }^{3}$, Mohamed Najib Mohamed Unni ${ }^{4}$, Ashley Edward Roy Soosay ${ }^{2}$, Henry Rantai Gudum ${ }^{2}$, Elizabeth George ${ }^{5}$, Jin Ai Mary Anne Tan \\ ${ }^{I}$ University of Malaya, Kuala Lumpur, Malaysia \\ 2 Universiti Malaysia Sarawak, Sarawak, Malaysia \\ ${ }^{3}$ Sarawak General Hospital, Sarawak, Malaysia \\ ${ }^{4}$ Hospital Miri, Sarawak, Malaysia \\ ${ }^{5}$ Universiti Putra Malaysia, Kuala Lumpur, Malaysia}

Introduction: Beta-thalassemia results in reduced or absence of $\beta$ globin chain synthesis and affected patients require monthly blood transfusions and iron chelation therapies for survival. Rapid characterization of the mutations is essential for genetic counseling and clinical management of thalassemia major children. This study aims to develop effective molecular screening techniques for $\beta$ thalassemia in Malaysia and to identify the types of $\beta$-thalassemia mutations in Sarawak patients as there is a paucity of data regarding thalassemia in East Malaysia. Methods: A total of 40 blood samples were collected from $\beta$-thalassemia patients in Sarawak. Quantitative real-time PCR (qPCR) using in-house developed high resolution melting (HRM) analysis and TaqMan genotyping assays were used to screen for the $\beta$-mutations. Established molecular techniques were used to confirm the results of qPCR and also to screen for rare mutations in the patients. Results: HRM analysis identified homozygosity and heterozygosity for the Filipino deletion in 21 and 12 patients respectively. HRM analysis detected a high frequency of the Filipino deletion $(82.5 \%)$ in thalassemia patients in Sarawak. Seven common $\beta$-mutations (CD41/42, IVS2-654, HbE, CD17, IVS1-1, CD71/72, Poly A) and 2 rarer mutations (CD19, -29) were also identified using TaqMan genotyping assays and Amplification Refractory Mutation System. Confirmation of the HRM analysis and TaqMan genotyping assays with conventional techniques showed $100 \%$ sensitivity and specificity. Conclusion: Rapid, sensitive and specific assays are paramount to implementation of successful screening programs for thalassemia. The HRM analysis requires 2 hours while the TaqMan genotyping assays require only 1 hour and avoids post-PCR handling.

\section{MULTIPLE ACYL-COA DEHYDROGENASE DEFICIENCY PRESENTING AS FETAL POLYCYSTIC KIDNEY DISEASE IN THREE SIBLINGS}

Sulekha Rajagopalan, Bernadette Hanna, Alan Adno

Liverpool Hospital, Sydney, NSW, Australia

Multiple Acyl-CoA Dehydrogenase Deficiency (MADD) is an autosomal recessive condition affecting fatty acid, amino acid and choline metabolism. It occurs due to mutations in one of three known genes, ETFA, ETFB and ETFDH. We present a consanguineous family where three consecutive pregnancies were affected with MADD and report the radiological, biochemical and molecular findings. The three siblings died of Type 1 MADD which is the lethal neonatal-onset form of MADD associated with congenital organ anomalies. The first affected pregnancy was found to have large echogenic kidneys and decreased liquor on the 18 -week morphology ultrasound scan. The fetus died in-utero at 28 weeks gestation. Large echogenic kidneys and inferior vermis hypoplasia were identified during the second and third affected pregnancies. Both pregnancies resulted in neonatal death. The infants had enlarged polycystic kidneys with respiratory distress, hypotonia and metabolic acidosis. Routine newborn screening was suggestive of MADD in the second affected infant which was confirmed on a plasma acylcarnitine profile. Amniotic fluid acylcarnitines and fatty acid oxidation studies in the next affected pregnancy were consistent with MADD. Genetic testing confirmed homozygosity for a novel missense mutation in the ETFB gene in the two affected liveborn siblings from which DNA was available. The parents are keen to have pre-implantation genetic diagnosis to avoid recurrence of the disease in a future pregnancy.

\section{Hb FEILDING [BETA12(A9)Thr >Pro]: A NOVEL} UNSTABLE BETA GLOBIN CHAIN VARIANT

Tarn Donald $^{1,2}$, Nikhil Singh ${ }^{1,2}$, David Broad ${ }^{3}$, Peter Browett ${ }^{1,2}$, Neil Van de Water ${ }^{\prime}$

${ }^{1}$ Auckland District Health Board, Auckland, New Zealand

${ }^{2}$ LabPlus, Auckland, New Zealand

${ }^{3}$ Feilding Medical Centre, Manawatu, New Zealand

We report here a patient who was found to be heterozygous for a previously unreported beta chain variant. A 72-year-old Caucasian female, was found to have an abnormal hemoglobin as an incidental finding following $\mathrm{HbA} 1 \mathrm{C}$ analysis. Her full blood count revealed a mild normochromic anaemia with $\mathrm{Hb} 111 \mathrm{~g} / \mathrm{L}$ (115-150), MCV 93fL (80-100) and MCH 30pg (27-32). Isopropanol solubility tests and a variant $\mathrm{Hb}$ on HPLC comprising $37 \%$ of the total $\mathrm{Hb}$ suggested an unstable Haemoglobin variant.

We performed Sanger sequencing on seven overlapping amplicons covering the entire Beta globin gene, including the near promoter region and 3'UTR. We discovered the single base substitution, HBB:c.37A $>C$, which changes the codon for threonine at position 12 to a proline within the $\mathrm{N}$ terminal alpha helix (A) of the mature beta chain. We propose that this variant be called $\mathrm{Hb}$ Feilding [beta12(A9)Thr $>$ Pro]. Three dimensional modeling suggest minimal disruption of hemoglobin function; however, prolines introduced into helical structures have been reported to distort secondary structure and result in reduced solubility.

\section{DECISIONS ABOUT CONFIRMATORY PRENATAL TESTING FOLLOWING PREIMPLANTATION GENETIC DIAGNOSIS}

Tenielle Davis, Caroline Motteram, Luk Rombauts

Monash IVF, Melbourne, VIC, Australia

Preimplantation genetic diagnosis (PGD) is the process of genetically screening an embryo for either the correct chromosome complement or a specific inherited disorder to selectively transfer the genetically desired embryos to establish a healthy pregnancy. PGD allows the couple to be reassured from the beginning of the pregnancy that their child will be free of the familial genetic condition or will have a reduced risk of miscarriage due to aneuploidy. It also reduces 
the need for prenatal testing (chorionic villus sampling or amniocentesis) which is invasive and has a small associated miscarriage risk.

PGD is appealing and has many benefits over prenatal testing alone, however PGD results are not $100 \%$ accurate. PGD is a screening method and offers a 90-98\% confidence of the results. Subsequently Monash IVF routinely recommends confirmatory prenatal testing to all PGD couples. Best practice guidelines established by the European Society of Human Reproduction and Embryology (ESHRE) PGD Consortium (2004) recommend that IVF clinics make an effort to follow up with the parents after prenatal testing or birth, especially if confirmatory testing is not possible. Despite the recommendations from Monash IVF and ESHRE's PGD Consortium, the number of couples who achieve a pregnancy following PGD at Monash IVF that proceed with confirmatory prenatal testing is unknown. A self-administered survey will be sent couples who have had an established pregnancy following PGD at Monash IVF. We anticipate that $<20 \%$ of couples will have confirmatory prenatal testing and believe it be the first report of its kind in the literature.

\section{CROSS-CULTURAL GENETIC COUNSELING}

\section{EXPERIENCES IN HONG KONG, CHINA}

\author{
Tiong Yang Tan', Brian Chung ${ }^{2}$ \\ ${ }^{1}$ Victorian Clinical Genetics Services, Melbourne, VIC, Australia \\ ${ }^{2}$ Queen Mary Hospital, Hong Kong
}

Cultural competence of health professionals is becoming increasingly important in the Australasian socio-cultural milieu. While the first wave of immigrants hailed from an Anglo-Saxon background, subsequent waves have been from other European backgrounds, with Asian and African immigrants being the most recent additions to the Australian landscape. Cultural competence is not limited to being sensitive and empathetic towards the psychosocial needs of a patient from a different background, but involves enlightened examination of the counselor's own culture, the 'yardstick' against which other cultures are measured. Compassionate comprehension, by improving cultural knowledge, but not using it to generalize and stereotype the values, character and needs of individuals, should be an aim of all culturally competent counselors. Negative counseling experiences may arise from cultural and ethnic differences between counselor and counselee if the differences inhibit communication. We present several observational vignettes to highlight the cultural differences and similarities of patients presenting for clinical genetic counseling in Hong Kong, a Special Administrative Region of China. Our perspectives as Asian-born clinical geneticists with 'Western' training provide unique insights as well as challenges.

\section{SCREENING N-GLYCAN BIOMARKERS AND EXPLORING SUBOPTIMAL HEALTH AS A PREVENTIVE TOOL FOR SHS}

Wei Wang $^{1,2,3}$, Yuxiang Yan ${ }^{3}$

${ }^{1}$ Edith Cowan University, Perth, WA, Australia

${ }^{2}$ Beijing Municipal Key Laboratory of Clinical Epidemiology, Beijing, China

${ }^{3}$ Capital Medical University, Beijing, China

Suboptimal health status (SHS) is characterized by ambiguous health complaints, general weakness, and lack of vitality, and it has become a new public health challenge in China. SHS is believed to be a subclinical, reversible stage of chronic disease. As studies of intervention and prognosis for SHS are expected to become increasingly important, a reliable and valid instrument for its assessment is essential. A questionnaire for measuring SHS in urban Chinese was developed based on focus group discussions and a literature review. Questionnaire validity and reliability were eval- uated in a cross-sectional study of 3000 individuals. The analyses included tests for reliability and internal consistency, exploratory and confirmatory factor analysis, and tests for discriminative ability and convergent validity. The final questionnaire incorporated 25 items on SHS (SHSQ-25), and encompassed 5 subscales: fatigue, cardiovascular system, digestive tract, immune system, and mental status. The SHSQ-25 has proved to be a reliable and valid instrument for measuring subhealth status in urban Chinese. The progress of a combined genomics and glycomics study for screening biomarkers and exploring SHS as a preventive tool for non-communicable disease control and management will be presented.

\section{FIRST AUSTRALIAN FAMILY WITH DISTAL ARTHROGRYPOSIS TYPE 5D; COMPOUND HETEROZYGOUS MUTATIONS IN ECEL1 IDENTIFIED BY WHOLE EXOME SEQUENCING}

\author{
Christopher Barnett ', Emily Todd ${ }^{2}$, Royston Ong ${ }^{2}$, Mark Davis ${ }^{3}$, Richard \\ Allcock $^{4}$, Nigel Laing ${ }^{2}$, Gianina Ravenscroft ${ }^{2}$ \\ 'Women's and Children's Hospital, SA Pathology, Adelaide, SA, Australia \\ ${ }^{2}$ WA Institute for Medical Research and the Centre for Medical Research, Perth, WA, \\ Australia \\ ${ }^{3}$ Department of Anatomical Pathology, Royal Perth Hospital, Perth, WA, Australia \\ ${ }^{4}$ Lotterywest State Biomedical Facility Genomics at University of Western Australia, \\ WA, Australia
}

Distal arthrogryposis (DA) comprises a group of disorders in which congenital contractures occur, typically affecting the hands, feet, wrists, and ankles. The genetic basis for five forms of DA has been determined, most recently DA type 5D in which mutations in endothelin converting enzyme-like 1 (ECEL1) were identified. Here we present the first Australian case of DA 5D with compound heterozygous ECEL1 mutations identified using whole exome sequencing (WES).

The proband, a 5-year-old girl born to non-consanguineous Australian Caucasian parents, was born with extended hips, a dislocated right hip, fixed flexion contractures of the knees, adducted thumbs, marked epicanthic folds and bilateral ptosis. Pterygia at several sites were present. Cognitive development is normal. Morphology ultrasound during the couple's next pregnancy was suggestive of a recurrence. At birth this infant had a right ptosis, bilateral clubfeet, extended knees, pterygia and fixed flexion deformities of the fingers. The prominent pterygia and ptosis led to consideration of multiple pterygium syndrome. Sanger sequencing of CHRNA1 and CHRNG was normal. WES of the proband revealed compound heterozygosity for two novel mutations (c.1531G > A; p.Gly511Ser and c.1797-1G $>$ A) in a recently (2013) identified disease gene (ECEL1) for DA type 5D. The clinical features of the proband and affected sibling are in keeping with the clinical picture for DA type 5D.

The emergence of WES has led to the identification of disease genes from far fewer patient/family resources than previously possible. Arthrogryposis/fetal akinesia is one such disease group where WES is revolutionizing our ability to diagnose patients.

\section{ANDERSEN-TAWIL SYNDROME PRESENTING AS CATECHOLAMINERGIC POLYMORPHIC VENTRICULAR TACHYCARDIA (CPVT)}

\author{
Julie McGaughran', Paul Martin², Lauren Hunt ${ }^{1,2}$, John Atherton ${ }^{2}$ \\ ${ }^{1}$ Genetic Health Queensland, Brisbane, QLD, Australia \\ 2 Department of Cardiology, RBWH, Brisbane, QLD, Australia \\ Andersen-Tawil syndrome (ATS) is characterized by a triad \\ of episodic flaccid muscle weakness (i.e., periodic paralysis),
}


ventricular arrhythmias and prolonged QT interval, and dysmorphic features and short stature. KCNJ2, encoding the inward rectifier potassium channel 2 protein (Kir2.1), is the only gene in which mutations are known to cause ATS. Approximately $60 \%$ of individuals with ATS have a detectable mutation in KCNJ2.

The proband was referred at 32 years with a working diagnosis of CPVT. She described palpitations from the age of 13 years and had suffered two prior syncopal episodes. During pregnancy, she suffered recurrent runs of non-sustained bidirectional ventricular tachycardia requiring prolonged hospitalization in the final trimester. She had a normal echocardiogram and cardiac MRI. There was no family history of premature sudden death. Testing of the RYR2 hot spots was undertaken and no mutation found. Her daughter was under cardiac review and noted to be short and have some mild dysmorphic features. The diagnosis of Andersen-Tawil syndrome was considered. A novel mutation c. $434 \mathrm{~A}>\mathrm{G}$ was found in KCNJ2. This case highlights the need to consider AndersenTawil syndrome in a patient presenting with clinical features of CPVT

\section{AN AUSTRALIAN FAMILY WITH A NOVEL HETEROZYGOUS SMAD3 MUTATION INVOLVING THE MH1 DOMAIN}

\author{
Kathy Wu', Katherine Holman ${ }^{2}$, Bruce Bennetts ${ }^{2}$, David Sillence ${ }^{3}$ \\ ${ }^{1}$ Discipline of Genetic Medicine, Sydney Medical School, Sydney, NSW, Australia \\ 2 Department of Molecular Genetics, Children's Hospital at Westmead, Sydney, NSW, \\ Australia \\ ${ }^{3}$ Department of Clinical Genetics, Children's Hospital at Westmead, Sydney, NSW, \\ Australia
}

Objectives: Mutations in SMAD3 have recently been shown to cause aneurysm-osteoarthritis syndrome (AOS) or Loeys-Dietz syndrome type III. We report the clinical and radiological features as well as molecular genetics in an Australian family, with a novel SMAD3 mutation; and compare these with previously published reports. Methods: Case report and molecular genetic testing. Results: The proband presented at age 8 years with a joint hypermobility syndrome, and was subsequently diagnosed with Ehlers-Danlos syndrome of a Hypermobility type. Her clinical history included myopia, and osteopenia noted at age 17 years. At age 18 years, her height was on 75-90th percentile, with reduced upper:lower segment ratio, malar hypoplasia, joint hypermobility with Beighton score $8 / 9$, bilateral pes planus, lumbar scoliosis, soft/velvety/mildly translucent skin, and striae. TGFBR1 and TGFBR2 mutational analyses were normal. SMAD3 testing revealed a novel heterozygous mutation involving the MH1 domain, c.368A $>$ G (p.Asn123Ser). In silico analyses suggest that this variant is likely to be pathogenic. Family cascade testing showed that her 56-year-old father, who has a history of premature arthropathy, chronic atrial fibrillation, inguinal hernia repair, and ruptured appendix, was found to be a carrier of this SMAD3 mutation. Testing in other family members is awaited. Whole-body arterial surveillance at baseline was normal in both father and daughter. Conclusion: To date this previously unreported variant is associated in this family with a predominantly skeletal phenotype. Other variants in the MH1 domain were previously reported to be associated with both thoracic and abdominal aortic aneurysms. Ongoing arterial surveillance was recommended in this family.

\section{APPLICATION OF EXOME SEQUENCING FOR DETECTION OF MUTATIONS IN LEBER CONGENITAL \\ AMAUROSIS}

Ivan Prokudin', Yiran Guo ${ }^{2}$, Maree Flaherty ${ }^{3}$, John Grigg ${ }^{3}$, Brendan Keating ${ }^{2}$,

Hakon Hakonarson ${ }^{2}$, Robyn Jamieson ${ }^{3}$

${ }^{1}$ Children's Medical Research Institute, Sydney, NSW, Australia

${ }^{2}$ The Children's Hospital of Philadelphia, Philadelphia, PA, USA

${ }^{3}$ The Children's Hospital at Westmead, Sydney, NSW, Australia

Leber congenital amaurosis (LCA) is the most severe form of retinal degeneration causing partial or complete blindness within the first year of life. LCA is usually inherited in an autosomal recessive manner and is characterized by marked genetic heterogeneity with at least 19 known causative disease genes. Until recently, strategies for identification of causative mutations have been based on screening of particular gene segments and examination for known variants. Recent advances indicate approaches based on targeted next-generation sequencing (NGS) or whole exome sequencing are attractive options for disease gene identification in this disorder. We reviewed our cohort of LCA patients where application of selected conventional direct PCR sequencing and screening for known pathogenic polymorphisms identified mutations in 8 of 16 families including in RPE65, CEP290, CRB1, and GUCY2D. In one patient where testing in parts of the LCA genes did not reveal any mutations, whole exome sequencing was undertaken which revealed a novel homozygous mutation in one of the known LCA genes, TULP1, not previously included in the LCA screening panel. Further work is underway to determine the proportion of our other cases that may be resolved using NGS. This work indicates that NGS provides an important clinical advance for genetic diagnosis for patients with heterogeneous retinal disorders such as LCA.

\section{NOVEL PHENOTYPIC FEATURES ASSOCIATED WITH INCREASED DOSAGE OF SOX3}

Tiong Yang Tan ${ }^{1}$, Bert Shugg ${ }^{2}$, Peter Farlie ${ }^{3}$, Trent Burgess ${ }^{4}$

1 Victorian Clinical Genetics Services, Parkville, VIC, Australia

${ }^{2}$ University of Tasmania, Hobart, TAS, Australia

${ }^{3}$ Murdoch Children's Research Institute, Parkville, VIC Australia

${ }^{4}$ Victorian Clinical Genetics Services, Parkville, VIC Australia

SOX3 belongs to the Sry-related-HMG-box-containing group of transcription factors critical in vertebrate development. SOX3 plays a key role in the development of the hypothalamic-pituitary axis and gonadal differentiation. Disruption of SOX3, either by direct involvement or copy number variation of its regulatory region, has been implicated in several human conditions. Duplications of chromosome Xq27.1 involving the SOX3 gene result in X-linked panhypopituitarism in males, with some phenotypic variability depending on the extent of the copy number change. Additionally, duplications of the regulatory region surrounding SOX 3 have been observed in patients with XX male sex reversal. We present a boy with partial absence of the corpus callosum, dyslalia, mild developmental delay, divergent strabismus and bilateral retinal colobomas associated with a complex duplication/triplication of chromosome Xq27.1 involving only the SOX3 gene. His mother, a carrier of the SOX3 change, has normal intellect and development, but also had bilateral retinal colobomas and mild dyslalia. This is the first time a CNV involving SOX3 has been reported with an ocular phenotype. This may suggest that ocular development is more tolerant of SOX3 dosage changes than the hypothalamus and pituitary gland. Our data are consistent with Sox 3 transgenic animal models and underscores the importance of Sox 3 dosage in the development of the brain, eyes and hypothalamic-pituitary axis. 